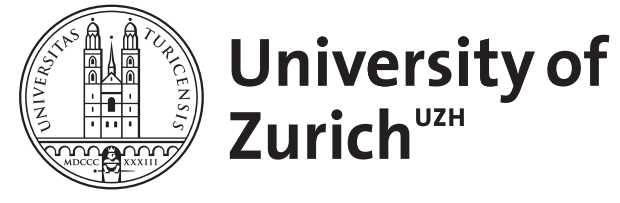
Archive

University of Zurich

University Library

Strickhofstrasse 39

CH-8057 Zurich

www.zora.uzh.ch

Year: 2017

\title{
Activation mechanism of the calcium-activated chloride channel TMEM16A revealed by cryo-EM
}

Paulino, Cristina ; Kalienkova, Valeria ; Lam, Andy K M ; Neldner, Yvonne ; Dutzler, Raimund

DOI: https://doi.org/10.1038/nature24652

Posted at the Zurich Open Repository and Archive, University of Zurich

ZORA URL: https://doi.org/10.5167/uzh-144591

Journal Article

Accepted Version

Originally published at:

Paulino, Cristina; Kalienkova, Valeria; Lam, Andy K M; Neldner, Yvonne; Dutzler, Raimund (2017). Activation mechanism of the calcium-activated chloride channel TMEM16A revealed by cryo-EM. Nature, 552(7685):421-425.

DOI: https://doi.org/10.1038/nature24652 


\section{Activation mechanism of the calcium-activated chloride channel TMEM16A revealed by cryo-EM}

Cristina Paulino ${ }^{1 *}$, Valeria Kalienkova ${ }^{1}$, Andy K. M. Lam ${ }^{1}$, Yvonne Neldner ${ }^{1}$ and Raimund Dutzler $^{1}$

${ }^{1}$ Department of Biochemistry University of Zurich, Winterthurer Str. 190, CH-8057 Zurich, Switzerland

* Present Address, Department of Structural Biology at the Groningen Biomolecular Sciences and Biotechnology Institute, University of Groningen, Nijenborgh 7, 9747 AG Groningen, The Netherlands 
The calcium-activated chloride channel (CaCC) TMEM16A is a ligand-gated anion channel that opens in response to the increase of the intracellular $\mathrm{Ca}^{2+}$ concentration ${ }^{1-3}$. The protein is widely expressed ${ }^{4}$ and contributes to diverse physiological processes such as transepithelial chloride transport and the control of electrical signaling in smooth muscles and certain neurons ${ }^{5-7}$. As member of the TMEM16 (or anoctamin) family of membrane proteins, TMEM16A is closely related to paralogs that function as scramblases, which facilitate the bidirectional movement of lipids across membranes ${ }^{8-11}$. The unusual functional diversity of the TMEM16 family and the relationship between two seemingly incompatible transport mechanisms has been the focus of recent investigations. Previous breakthroughs were obtained from the X-ray structure of the fungal lipid scramblase nhTMEM16 ${ }^{12,13}$, and from the cryo-electron microscopy (cryoEM) structure of mouse TMEM16A (mTMEM16A) at $6.6 \AA^{14}$. Whereas the latter structure disclosed the architectural differences that distinguish ion channels from lipid scramblases, its low resolution neither permitted a detailed molecular description of the protein nor did it provide any insight into its activation by $\mathrm{Ca}^{2+}$. To address these questions we here describe the structures of mTMEM16A at high resolution in the presence and absence of $\mathrm{Ca}^{2+}$. Both structures reveal the differences between ligandbound and ligand-free states of a $\mathrm{CaCC}$ and, combined with functional experiments, they suggest a mechanism for gating. During activation, the binding of $\mathrm{Ca}^{2+}$ to a site located within the transmembrane domain in vicinity of the pore alters its electrostatics and triggers a conformational rearrangement of an $\alpha$-helix that comes into physical contact with the bound ligand and thus directly couples ligand binding to pore opening. Our study describes a process that is unique among channel proteins but that is presumably general for both functional branches of the family. 
We have purified mTMEM16A in the absence and presence of $\mathrm{Ca}^{2+}$, confirmed its functional integrity by reconstitution (Extended Data Fig. 1a, b), and determined structures for each condition by single-particle cryo-EM (Fig. 1, Extended Data Figs. 1 and 2). The respective resolutions of the EM maps of the $\mathrm{Ca}^{2+}$-bound and $\mathrm{Ca}^{2+}$-free protein are 3.75 and $4.06 \AA$ with large parts of the transmembrane domain being substantially better resolved (Extended Data Figs. 1g, h and 2f, g). Both datasets are of high quality and define the architecture of the channel in distinct states (Fig. 1, Extended Data Figs. 3-5, Supplementary Videos 1 and 2). The refined $\mathrm{Ca}^{2+}$-bound and $\mathrm{Ca}^{2+}$-free structures display conformations that are very similar over the majority of the protein except for changes in the region surrounding the $\mathrm{Ca}^{2+}$-binding sites (Fig. 1, Extended Data Figs. 3a and 6a, b, Supplementary Video 3).

The $\mathrm{Ca}^{2+}$-bound structure brings into focus molecular details that have been postulated based on low resolution data ${ }^{14}$ (Fig. 1). The protein is a homodimer that, with respect to its general architecture, resembles the lipid scramblase nhTMEM16 (Extended Data Fig. 6c, d). Each subunit contains cytosolic N- and C-terminal domains, a transmembrane unit consisting of ten membrane-spanning $\alpha$-helices, and an extracellular component (Extended Data Fig. 5). The comparably small dimer interface burying only $1006 \AA^{2}$ (i.e. $1.3 \%$ ) of the combined molecular surface is formed exclusively by interactions between residues located at the outer part of $\alpha$-helix 10 (Extended Data Fig. 5d). Below the contact region, the separation of both symmetry-related helices opens up a large membrane accessible cavity that is presumably filled with lipids. On the extracellular side, long stretches of amino acids connecting $\alpha$-helices 1-2, 5-6 and 9-10, interact to form a folded domain consisting of coil regions with interspersed elements of secondary structure that are stabilized by four disulfide bridges (Extended Data Figs. $3 b$ and 5e). On the intracellular side, the N-terminal domain displays a ferredoxin-like fold that resembles the equivalent part in the scramblase nhTMEM16 (ref. 13, Extended Data Fig. 5f), whereas the preceding 115 amino acids, which are only present in the 
$a$ isoforms of the channel ${ }^{1}$, are not defined in the mTMEM16A structure. Close to the dimer axis, the N-terminal domain participates in an intra-subunit interaction with the sole structured part of the cytoplasmic C-terminus, helix Cal (Fig. 1, Extended Data Figs. 5b and 6a). The same location harbors the poorly defined and thus presumably inherently flexible extended $\alpha 2-\alpha 3$ loop that also contains a short $\alpha$-helical region ( $\alpha 2$ ', Extended Data Figs. 1f, 2 d, $4 \mathrm{a}$ and 5a, b, g). This loop carries an insertion in $c$ splice variants ${ }^{1}$, with mutations influencing the activation properties of the channel ${ }^{15-17}$ (Extended Data Fig. 5a, g).

The ion conduction pore of TMEM16A is contained within each subunit and is formed by $\alpha-$ helices 3-7 (Fig. 2a). Its shape resembles an hourglass with a small extracellular and a large intracellular vestibule, which are bridged by a narrow, about $20 \AA$ long neck region (Fig. 2ad). Since the pore is continuous, the observed conformation is presumably close to a conducting state. However, with a diameter of only $2.5 \AA$ at its constriction, it would have to expand to accommodate permeating anions, whose size ranges between 3.6 and $4.1 \AA$ in case of $\mathrm{Cl}^{-}$and $\mathrm{I}^{-}$, respectively (Extended Data Fig. 7a, b). Such dilation could be attained by the movement of the inherently flexible $\alpha$-helix 3 (Extended Data Figs. 1f, 2d, e) and local changes of side-chain conformations. Both cone-shaped vestibules are highly hydrophilic and are lined by ionizable residues (Fig. 2b, d). The excess of basic amino acids confers a positive electrostatic environment throughout the pore (Extended Data Fig. 8a, b) thus lowering the energy barrier for anion conduction ${ }^{14}$. The extracellular vestibule is on one side delimited by the $\alpha 1-\alpha 2$ and $\alpha 5-\alpha 6$ loops and on the opposite side by the mobile region connecting $\alpha-$ helices 3 and 4 (Fig. 2a). The $\alpha 5-\alpha 6$ loop contains residues that were previously proposed to be part of the pore-lining region ${ }^{18,19}$ (Fig. 2b). In contrast to the vestibules, the narrow neck is amphiphilic and does not contain charged amino acids (Fig. 2c). It is confined by $\alpha$-helices $3-$ 6 on its outer and 4-7 on its inner half (Fig. 2a, c). The narrow conduit is shielded from the membrane by extended interactions between $\alpha$-helices 4 and 6 , which are detached in the 
scramblase nhTMEM16 (ref. 14, Extended Data Figs. 6c and 7c). Below the neck, $\alpha$-helices 4 and 6 separate, thereby generating a spacious intracellular vestibule (Fig. 2a, d, Extended Data Fig. 7a, b). This separation opens up a gap that might be partly accessible to the lipid bilayer and provides space for the rearrangement of $\alpha$-helix 6 in the $\mathrm{Ca}^{2+}$-free state (Extended Data Fig. $7 \mathrm{a}, \mathrm{d}, \mathrm{g})$. At the surface of the wide intracellular vestibule, conserved residues of $\alpha$ helices 6-8 form a $\mathrm{Ca}^{2+}$-binding site (Fig. 2e). This site is well defined in our experimental data, except for the carboxylate groups of interacting acidic residues, which are frequently not detected in cryo-EM densities (Extended Data Fig. 4b). Distinct peaks reveal the presence of two $\mathrm{Ca}^{2+}$ ions residing at equivalent locations as in nhTMEM16 (ref. 12, Fig. 2e). Analogously, both ions are coordinated by five acidic residues (Fig. 2e), all of which have been previously described to affect $\mathrm{Ca}^{2+}$ activation of mTMEM16A $\mathrm{A}^{12,18,20}$. In contrast to nhTMEM16, where a single asparagine on $\alpha 6$ contributes to the coordination of the upper $\mathrm{Ca}^{2+}$, the corresponding $\mathrm{Ca}^{2+}$ ion in mTMEM16A interacts with three polar residues (Asn 650, Asn 651 and, slightly further apart, Asn 730, Fig. 2e) whose mutation to alanine lowers the potency of $\mathrm{Ca}^{2+}$ (ref 12, Extended Data Fig. 8e-g). An additional interaction, although at a somewhat larger distance, is provided by the unpaired main-chain carbonyl of Gln 646 (Fig. 2e, Extended Data Fig. 4b). The comparison of the channels TMEM16A and B to other family members reveals an insertion of a single residue in this region (Extended Data Fig. 5c), which leads to a partial unwinding of $\alpha 6$. The energetic penalty caused by the hereby introduced $\pi$-helix bulge is probably stabilized by interactions with the bound ligand.

Cryo-EM data obtained for mTMEM16A purified in the absence of $\mathrm{Ca}^{2+}$ define the structure of the protein in a ligand-free state (Extended Data Figs. 2 and 3). In this structure, significant changes compared to the $\mathrm{Ca}^{2+}$-bound conformation are confined to the pore region (Figs. 1, 3a-c and Extended Data Fig. 6a, b, Supplementary Video 3 and 4). In contrast to the ligandbound structure, no density attributable to ions is found in the $\mathrm{Ca}^{2+}$-binding site (Extended 
Data Fig. 4c). Whereas comparably small differences are also observed in the narrow neck region for $\alpha$-helices 3,4 and 6 , the most pronounced conformational changes concern the inner half of $\alpha 6$ (Extended Data Figs. $6 \mathrm{a}, \mathrm{b}$ and $7 \mathrm{~d}-\mathrm{g}$ ). Upon release of $\mathrm{Ca}^{2+}$ from its binding site, this part of the helix has detached from its position in the $\mathrm{Ca}^{2+}$-bound state where it closely interacted with $\alpha$-helix 7 (Fig. 3a, b, Extended Data Fig. 7g). Its movement towards $\alpha 4$ closes the gap between both helices observed in the $\mathrm{Ca}^{2+}$-bound state at the intracellular part of the pore. At the same time, it opens a spacious, likely water-filled access pathway to the $\mathrm{Ca}^{2+}$-binding site (Fig. 3b, Extended Data Fig. 7g). The rearrangement of $\alpha 6$ can be approximated by a hinge movement around Gly 644 along with its unfolding at positions proximal to Gly 656, which itself moves about $15 \AA$ (Fig. 3a, Extended Data Fig. 6b, Supplementary Video 4). The remainder of the helix is poorly defined in the EM density and thus presumably mobile (Extended Data Fig. 4c). The movement around Gly 644 is accompanied by the relaxation of the strained $\pi$-helix and the formation of a canonical $\alpha$-helix (Fig. 3c). Although the movement of $\alpha 6$ does not lead to an occlusion of the intracellular vestibule, it narrows the neck of the pore, thus potentially affecting the entry of permeating ions (Extended Data Fig. $7 \mathrm{~d}-\mathrm{g}$ ). Of note is the fact that the absence of $\mathrm{Ca}^{2+}$ renders the electrostatic environment in this region negative, which in turn increases the barrier for anion conduction (Fig. 3d, Extended Data Fig. 8c, d).

To further characterize the role of Gly 644 for channel activation, we have replaced it by amino acids with reduced conformational flexibility and investigated activation by electrophysiology. Since the residue is not directly involved in ligand interactions, we expect mutations at this position to disturb the equilibrium between open and closed conformations $^{21,22}$. This is observed for the mutations G644A and G644P, which both cause a left-shift in the $\mathrm{EC}_{50}$ (Fig. 4a, b, Extended Data Fig. 9a, b). In the latter case, significant current in the absence of $\mathrm{Ca}^{2+}$ indicates basal activity of the mutant, which is not observed in 
macroscopic recordings of WT (Fig. 4b, Extended Data Figs. 8e and 9b). These basal currents are strongly outwardly rectifying (Extended Data Fig. 9c), consistent with an electrostatic barrier at the location of the vacant binding site which impedes anion permeation from the cytoplasm (Fig. 3d, Extended Data Fig. 8d). The movement of $\alpha 6$ and the accompanying change in its environment is also consistent with the functional behavior of mutants at positions not involved in $\mathrm{Ca}^{2+}$ binding that are distant from the hinge. Whereas changes in the $\mathrm{EC}_{50}$ were small for mutations of Gly 656 , which forms the pivot for the unfolding of the ensuing part of the helix, decreased potency was observed for mutations of the conserved Pro 658 located a helix turn further towards the cytoplasm (Fig. 4c, d, Extended Data Figs. 5c, 9d-g). Next, we were interested in whether the transition into a closed state upon the release of $\mathrm{Ca}^{2+}$ impedes the access to the intracellular vestibule. We have therefore monitored the state-dependent modification of residues by thiol-reactive reagents. The time dependence of modification of a cysteine introduced at the position of Lys 588, which is located deep in the vestibule close to the intracellular entrance to the neck, are very similar in the presence or absence of $\mathrm{Ca}^{2+}$, indicating that the accessibility of this position is not significantly affected by channel closure as predicted from the structure (Fig. 4e-g, Extended Data Figs. 7g and 9h, i). In contrast, the corresponding mutation for Ser 592, which is located one helix turn towards the extracellular side (Fig. 4e, h, Extended Data Fig. 9i-1) is not modified, reflecting the inaccessibility of the site to MTS reagents due to the restricted geometry of the neck. Nevertheless, we expect changes in this narrow region to play an important role in gating as evidenced by the pore mutation I550A, which increases the potency of $\mathrm{Ca}^{2+}$ and gives rise to basal currents (Figs. 4e, i, Extended Data Fig. 9m).

Collectively our data have provided detailed insight into the mechanism of activation of mTMEM16A, which occurs independently in each subunit of the dimeric protein ${ }^{23,24}$. During this process, the inner half of $\alpha$-helix 6 acts as a gating element (Fig. 5). In the closed channel, 
the helix is in a relaxed state, allowing access of $\mathrm{Ca}^{2+}$ from the cytoplasm to the vacant ligand-binding site. Binding of presumably two $\mathrm{Ca}^{2+}$ ions to four acidic residues on $\alpha$-helices 7 and 8 precedes pore opening (Figs. $3 b$ and 5a). The resulting high positive charge density creates an attractive interaction platform for residues located on the distant $\alpha$-helix 6 . This is sufficient to shift the conformational equilibrium of the helix to a position where it makes stabilizing contacts with $\alpha$-helices 7 and 8 as defined by the $\mathrm{Ca}^{2+}$-bound structure. The concurrent reorientation of Glu 654 upon interaction with the bound ligand causes the formation of a $\pi$-helix, which is stabilized by the interaction of the unpaired backbone carbonyl of Gln 646 with the upper $\mathrm{Ca}^{2+}$ ion (Figs. 3c and 5b). The energetic penalty associated with this conformational transition is reflected in the stability of the closed state in the absence of ligand, as manifested in the undetectable basal activity of the channel. The conformational transition during activation closes the aqueous path to the $\mathrm{Ca}^{2+}$-binding site consistent with the stronger voltage dependence observed during channel closing compared to opening $^{15,25}$. In contrast to the dynamic lower part, the upper pore region forms a narrow conduit whose structural changes appear to be comparably small during activation. Nevertheless, we think that small conformational changes within the neck constricting the channel (Extended Data Fig. 7e-g) combined with the presence of an electrostatic barrier in absence of $\mathrm{Ca}^{2+}$ (Fig. 3d, Extended Data Fig. 8d), both contribute to the impediment of anion permeation in the ligand-free state. In the open state, the neck region provides a favourable environment for selective ion permeation. Anions, which have presumably shed most of their hydration shell, interact with residues of an amphiphilic pore whereas positively charged sidechains located at both ends contribute to an attractive electrostatic environment (Extended Data Fig. 8b) without creating localized strong binding sites ${ }^{14,26}$ thus accounting for the strong selectivity for anions over cations ${ }^{14,23,24,27}$. In this narrow conduit, polar residues compensate for the removal of coordinating water molecules, whereas hydrophobic residues increase the energetic penalty for smaller ions, consistent with the observed lyotropic permeability 
sequence, which favors larger anions ${ }^{26,28}$. Ion-protein interactions in the neck might also account for the observed stabilization of the open state by permeating ions ${ }^{15,29,30}$. Due to the conservation of the $\mathrm{Ca}^{2+}$-binding site and of other sequence elements located on $\alpha 6$, it can be assumed that activation proceeds by a similar mechanism in lipid scramblases of the TMEM16 family ${ }^{1-13}$.

In summary, our work describes an activation process for TMEM16A that is distinct from other ligand-gated ion channels. Instead of binding to a separate structural unit, the ligand in TMEM16A directly interacts with the pore region, thereby immobilizing a transmembrane helix involved in gating and influencing ion conduction by altering the electrostatic properties of the pore.

1 Caputo, A. et al. TMEM16A, a membrane protein associated with calcium-dependent chloride channel activity. Science 322, 590-594 (2008).

2 Yang, Y. D. et al. TMEM16A confers receptor-activated calcium-dependent chloride conductance. Nature 455, 1210-1215 (2008).

3 Schroeder, B. C., Cheng, T., Jan, Y. N. \& Jan, L. Y. Expression cloning of TMEM16A as a calcium-activated chloride channel subunit. Cell 134, 1019-1029 (2008).

4 Huang, F. et al. Studies on expression and function of the TMEM16A calciumactivated chloride channel. Proc. Natl. Acad. Sci. USA 106, 21413-21418 (2009).

5 Huang, F., Wong, X. \& Jan, L. Y. International Union of Basic and Clinical Pharmacology. LXXXV: calcium-activated chloride channels. Pharmacol. Rev. 64, 115 (2012).

6 Oh, U. \& Jung, J. Cellular functions of TMEM16/anoctamin. Pflugers Arch. 468, $443-453$ (2016). 
7 Pedemonte, N. \& Galietta, L. J. Structure and Function of TMEM16 Proteins (Anoctamins). Physiol. Rev. 94, 419-459 (2014).

8 Suzuki, J. et al. Calcium-dependent phospholipid scramblase activity of TMEM16 protein family members. J. Biol. Chem. 288, 13305-13316 (2013).

9 Suzuki, J., Umeda, M., Sims, P. J. \& Nagata, S. Calcium-dependent phospholipid scrambling by TMEM16F. Nature 468, 834-838 (2010).

10 Whitlock, J. M. \& Hartzell, H. C. Anoctamins/TMEM16 Proteins: Chloride Channels Flirting with Lipids and Extracellular Vesicles. Annu. Rev. Physiol (2016).

11 Malvezzi, M. et al. Ca2+-dependent phospholipid scrambling by a reconstituted TMEM16 ion channel. Nat. Commun. 4, 2367, doi:10.1038/ncomms3367 (2013).

12 Brunner, J. D., Lim, N. K., Schenck, S., Duerst, A. \& Dutzler, R. X-ray structure of a calcium-activated TMEM16 lipid scramblase. Nature 516, 207-212 (2014).

13 Brunner, J. D., Schenck, S. \& Dutzler, R. Structural basis for phospholipid scrambling in the TMEM16 family. Curr. Opin. Struct. Biol. 39, 61-70 (2016).

14 Paulino, C. et al. Structural basis for anion conduction in the calcium-activated chloride channel TMEM16A. eLife 6, e26232, doi:10.7554/eLife.26232 (2017).

15 Xiao, Q. et al. Voltage- and calcium-dependent gating of TMEM16A/Ano1 chloride channels are physically coupled by the first intracellular loop. Proc. Natl. Acad. Sci. USA 108, 8891-8896 (2011).

16 Ferrera, L. et al. Regulation of TMEM16A chloride channel properties by alternative splicing. J. Biol. Chem. 284, 33360-33368 (2009).

17 Cruz-Rangel, S. et al. Gating modes of calcium-activated chloride channels TMEM16A and TMEM16B. J. Physiol. 593, 5283-5298 (2015).

18 Yu, K., Duran, C., Qu, Z., Cui, Y. Y. \& Hartzell, H. C. Explaining calcium-dependent gating of anoctamin-1 chloride channels requires a revised topology. Circ. Res. 110, 990-999 (2012). 
19 Cruz-Rangel, S. et al. Extracellular protons enable activation of the CalciumDependent chloride Channel TMEM16A (ANO1). J. Physiol. (2016).

20 Tien, J. et al. A comprehensive search for calcium binding sites critical for TMEM16A calcium-activated chloride channel activity. eLife, 3, e02772, doi:10.7554/eLife.02772 (2014).

21 Colquhoun, D. Binding, gating, affinity and efficacy: the interpretation of structureactivity relationships for agonists and of the effects of mutating receptors. $\mathrm{Br} . \mathrm{J}$. Pharmacol. 125, 924-947 (1998).

22 Auerbach, A. Thinking in cycles: MWC is a good model for acetylcholine receptorchannels. J. Physiol. 590, 93-98 (2012).

23 Lim, N. K., Lam, A. K. \& Dutzler, R. Independent activation of ion conduction pores in the double-barreled calcium-activated chloride channel TMEM16A. J. Gen. Physiol. 148, 375-392 (2016).

24 Jeng, G., Aggarwal, M., Yu, W. P. \& Chen, T. Y. Independent activation of distinct pores in dimeric TMEM16A channels. J. Gen. Physiol. 148, 393-404, doi:10.1085/jgp.201611651 (2016).

25 Arreola, J., Melvin, J. E. \& Begenisich, T. Activation of calcium-dependent chloride channels in rat parotid acinar cells. J. Gen. Physiol. 108, 35-47 (1996).

26 Qu, Z. \& Hartzell, H. C. Anion permeation in $\mathrm{Ca}(2+)$-activated $\mathrm{Cl}(-)$ channels. J. Gen. Physiol. 116, 825-844 (2000).

27 Terashima, H., Picollo, A. \& Accardi, A. Purified TMEM16A is sufficient to form Ca2+-activated Cl- channels. Proc. Natl. Acad. Sci. USA 110, 19354-19359 (2013).

28 Ni, Y. L., Kuan, A. S. \& Chen, T. Y. Activation and inhibition of TMEM16A calcium-activated chloride channels. PLoS One 9, e86734, doi:10.1371/journal.pone.0086734 (2014). 
29 Perez-Cornejo, P., De Santiago, J. A. \& Arreola, J. Permeant anions control gating of calcium-dependent chloride channels. J. Membr. Biol. 198, 125-133 (2004).

30 Betto, G. et al. Interactions between permeation and gating in the TMEM16B/anoctamin2 calcium-activated chloride channel. J. Gen. Physiol. 143, 703-718 (2014).

Supplementary Information is available in the online version of the paper.

\section{Acknowledgements}

We thank O. Medalia and M. Eibauer, the center for microscopy and image analysis (ZMB) of the University of Zurich, and the Mäxi foundation for the support and access to the Electron Microscopes. Justin D. Walter is acknowledged for comments on the manuscript and all members of the Dutzler lab for help at various stages of the project. This research was supported by a grant from the European Research Council (no 339116, AnoBest). C. P. was supported by a postdoctoral fellowship (Forschungskredit) of the University of Zurich.

\section{Author Contributions}

V.K. and Y.N. expressed and purified the protein for cryo-EM and functional reconstitution and performed the flux assay. C.P. prepared the sample for cryo-EM, collected EM data and proceeded with structure determination. A.K.M.L. generated mutants, performed electrophysiological recordings and fitted data. C.P., V.K., A.K.M.L., Y.N and R.D. jointly planned experiments, analyzed the data and wrote the manuscript.

\section{Author Information}


Reprints and permissions information is available at www.nature.com/reprints. The authors declare no competing financial interests. Correspondence and requests for materials should be addressed to R.D. (dutzler@bioc.uzh.ch).

Figure 1 | TMEM16A structures. Ribbon representation of a superposition of the $\mathrm{Ca}^{2+}-$ bound (green) and $\mathrm{Ca}^{2+}$-free (violet) structures of mTMEM16A. Selected $\alpha$-helices are labeled. The view is from within the membrane with the extracellular side at the top. Membrane boundaries are indicated. Subunits are shown in bright and dark shades of the same color. $\mathrm{Ca}^{2+}$ ions in the $\mathrm{Ca}^{2+}$-bound conformation are displayed as blue spheres.

Figure 2 | Pore and $\mathrm{Ca}^{2+}$-binding site in the $\mathrm{Ca}^{2+}$-bound structure. a, Ion conduction pore of the mTMEM16A subunit. Pore surface (probe diameter $2.2 \AA$ ) is shown as grey mesh. Sections of the pore are indicated, including: $\mathbf{b}$, the extracellular vestibule; c, the neck; and d, the intracellular vestibule. e, Structure of the $\mathrm{Ca}^{2+}$-binding site with EM density $(8 \sigma)$ of the two $\mathrm{Ca}^{2+}$ ions superimposed as mesh. The view is rotated by about $90^{\circ}$ compared to a. a-e, Protein is shown as $\mathrm{C} \alpha$-representation in unique colours, side-chains of selected residues as sticks, $\mathrm{Ca}^{2+}$ ions as blue spheres.

Figure 3 | $\mathrm{Ca}^{2+}$-free structure and conformational changes upon $\mathrm{Ca}^{2+}$ release. a, Superposition of $\alpha$-helices 3-8 of the $\mathrm{Ca}^{2+}$-bound (green) and $\mathrm{Ca}^{2+}$-free structure (violet). The view is rotated by $90^{\circ}$ around the dimer axis compared to Fig. $1 . \mathbf{b}, \mathrm{Ca}^{2+}$-binding site of the $\mathrm{Ca}^{2+}$-bound (left) and the $\mathrm{Ca}^{2+}$-free structure (right). c, Section of $\alpha 6$. Main chain atoms are shown with $\mathrm{H}$-bonds depicted as black and an interaction with $\mathrm{Ca}^{2+}$ as orange dashed lines. d, 
Difference in the electrostatic potential between the $\mathrm{Ca}^{2+}$-bound and $\mathrm{Ca}^{2+}$-free conformations along the pore region. Asterisk indicates the location of the $\mathrm{Ca}^{2+}$-binding site.

Figure 4 | Functional characterization of conformational changes. a, $\alpha$-helix 6 in the $\mathrm{Ca}^{2+}-$ bound (green) and $\mathrm{Ca}^{2+}$-free (violet) conformations. b, $\mathrm{Ca}^{2+}$ concentration-response relationships of the mutants G644A and G644P, c, G656A and G656P, and, d, P658A and P658G. e, Location of residues within the pore. Time-dependent modification of the mutant 6C-K588C with MTSEA, $\mathbf{f}$, in the presence and, $\mathbf{g}$, in the absence of $\mathrm{Ca}^{2+} . \mathrm{f}, \mathrm{g}$, Solid lines show a fit to a double-exponential function. $\mathbf{h}$, Same experiment as in $\mathrm{f}$ on mutant $6 \mathrm{C}$ K588Q/S592C. i, $\mathrm{Ca}^{2+}$ concentration-response relationship of the mutant I550A. b-d, i, Measurements are from excised inside-out patches at $80 \mathrm{mV}$. Data show mean values of normalized currents measured from either 6 (G644A, P658A), 7 (G644P, G656P, I550A) or 8 (G656A, P658G) biological replicates, errors are s.e.m. Solid lines show a fit to a Hill equation, WT (Extended Data Fig. 8e) is displayed as dashed line for comparison. f-h, Data show averages of either 7 (6C-K588Q/S592C), $8\left(6 \mathrm{C}-\mathrm{K} 588 \mathrm{C} \mathrm{Ca}^{2+}\right)$ or $9\left(6 \mathrm{C}-\mathrm{K} 588 \mathrm{C}\right.$ no $\left.\mathrm{Ca}^{2+}\right)$ biological replicates, errors are s.e.m.

Figure 5 | Activation mechanism. a, Schematic depiction of the activation of TMEM16A. Top panels are viewed from within the membrane, bottom panels from the intracellular side. Pore-lining helices are depicted as green cylinders, $\mathrm{Ca}^{2+}$ and $\mathrm{Cl}^{-}$as blue and red spheres. b, Conformational changes in $\alpha$-helix 6 upon $\mathrm{Ca}^{2+}$ binding. Views are as in a. Gly 644 (hinge) is shown as sphere, the side chain of Glu 654 as sticks. The position of Gln 646 is coloured in violet and its interaction with $\mathrm{Ca}^{2+}$ in the open state is indicated. Bound $\mathrm{Ca}^{2+}$ ions are shown as blue spheres. 


\section{METHODS}

\section{Protein expression and purification.}

HEK293 cells constitutively expressing mTMEM16A(ac) were cultivated and harvested as described previously ${ }^{14}$. To improve the sample quality, the duration of purification was significantly reduced from $27 \mathrm{~h}$ to $12 \mathrm{~h}$. All steps were carried out at $4{ }^{\circ} \mathrm{C}$. Cells from $23 \mathrm{lof}$ adhesion culture (in $230010 \mathrm{~cm}$ dishes) were resuspended in $230 \mathrm{ml}$ of buffer A $(20 \mathrm{mM}$ HEPES pH 7.5, $150 \mathrm{mM} \mathrm{NaCl}, 0.5 \mathrm{mM} \mathrm{CaCl}_{2}$ ) containing 1\% digitonin (AppliChem) and protease inhibitors (cOmplete, Roche). Membranes were solubilized by gentle agitation in batch for $2 \mathrm{~h}$. The sample was centrifuged at 22,000 g for $30 \mathrm{~min}$ and subsequently filtered through a $5 \mu \mathrm{m}$ filter (Minisart, Sartorius) to remove the insoluble fraction. The supernatant was applied to $3 \mathrm{ml}$ of streptavidin UltraLink resin (Pierce, ThermoScientific) and protein was bound to the beads by incubation in batch for $1.5 \mathrm{~h}$. The resin was subsequently washed with 60 column volumes of buffer B (20 mM HEPES pH 7.5, $150 \mathrm{mM} \mathrm{NaCl}, 0.5 \mathrm{mM} \mathrm{CaCl}$, $0.12 \%$ digitonin, Calbiochem). Protein was eluted with 3 column volumes of buffer B with 3 $\mathrm{mM}$ biotin (Sigma). Protein-containing fractions were deglycosylated for $2 \mathrm{~h}$ with PNGaseF, subsequently concentrated (100 kDa cut-off, Amicon Ultra) and applied to a Superose 6 column (GE-Healthcare) equilibrated in buffer B. The fractions from the main peak were pooled and concentrated yielding $70 \mu \mathrm{g}$ of pure mTMEM16A. The sample at a final concentration of $3.5 \mathrm{mg} \mathrm{ml}^{-1}$ was immediately used for cryo-EM sample preparation.

The purification of mTMEM16A in the absence of $\mathrm{Ca}^{2+}$ was performed as described above, except with the following buffer compositions: buffer $\mathrm{A}^{-\mathrm{Ca} 2+}(20 \mathrm{mM}$ HEPES pH 7.5, 150 $\mathrm{mM} \mathrm{NaCl}, 10 \mathrm{mM}$ EGTA) and buffer $\mathrm{B}^{-\mathrm{Ca} 2+}(20 \mathrm{mM}$ HEPES pH 7.5, $150 \mathrm{mM} \mathrm{NaCl}, 5 \mathrm{mM}$ EGTA, $0.12 \%$ digitonin, Calbiochem). All the buffers were prepared using calcium-free water for molecular biology (Millipore). Only plastic materials were used throughout the 
purification, and when needed pre-cleaned with $10 \mathrm{mM}$ EDTA pH 8.0 to minimize $\mathrm{Ca}^{2+}$ contamination. Purification from 191 of adhesion culture (in $190010 \mathrm{~cm}$ dishes) yielded 60 $\mu \mathrm{g}$ of pure mTMEM16A. The sample at a final concentration of $3.3 \mathrm{mg} \mathrm{ml}^{-1}$ was immediately used for cryo-EM sample preparation.

\section{Reconstitution and transport assays}

For reconstitution, mTMEM16A was purified from 101 of adhesion culture in the absence of $\mathrm{Ca}^{2+}$ as described above except that the concentration of EGTA was decreased to $1 \mathrm{mM}$ at the gel-filtration step. The reconstitution was performed as described ${ }^{34}$. Briefly, the liposomes were prepared as a 3:1 mixture of E. coli polar lipids/egg PC (Avanti polar lipids) and resuspended in reconstitution buffer (100 mM KCl, 10 mM HEPES pH 7.4, 1 mM EGTA). After detergent destabilization, the purified protein was added to the liposome suspension at a ratio of 1:75 (w/w). For the control sample, the equivalent amount of the protein buffer was added to the liposome suspension. The detergent was removed by addition of SM2 bio-beads (Bio-Rad). The ion flux assay was performed similarly to previous reports ${ }^{35}$. After harvesting, the buffer inside the liposomes was exchanged by freeze-thawing for assay buffer A (100 mM $\mathrm{Na}_{2} \mathrm{SO}_{4}, 10 \mathrm{mM}$ HEPES pH 7.4, 1 mM EGTA) containing either 0 or $1 \mu \mathrm{M}$ of free $\mathrm{Ca}^{2+}$. The liposomes were flash-frozen and stored at $-80{ }^{\circ} \mathrm{C}$ at a concentration of $10 \mathrm{mg} \mathrm{m}^{-1}$ until further use. On the day of the measurement, the liposomes were sonicated and diluted 50-fold into an assay buffer B (10 mM HEPES pH 7.4, 125 mM NaCl, $2 \mu \mathrm{M}$ ACMA, 1 mM EGTA) containing either 0 or $1 \mu \mathrm{M}$ free $\mathrm{Ca}^{2+}$. Fluorescence intensity was recorded at $5 \mathrm{~s}$ intervals on a TECAN Infinite M1000 plate reader, with $\lambda_{\mathrm{ex}}$ and $\lambda_{\mathrm{em}}$ of $410 \mathrm{~nm}$ and $490 \mathrm{~nm}$, respectively. The ion transport was initiated after $90 \mathrm{~s}$ by addition of the proton ionophore CCCP from a $100 \mu \mathrm{M}$ stock in DMSO to a final concentration of $1 \mu \mathrm{M}$. The data were normalized and displayed as $\mathrm{F} / \mathrm{F}_{\max }$. 


\section{Electron microscopy sample preparation and imaging}

$2 \mu 1$ of purified mTMEM16A at a concentration of $3.5 \mathrm{mg} \mathrm{ml}^{-1}$ in presence of $0.5 \mathrm{mM} \mathrm{CaCl}_{2}$ or at a concentration of $3.3 \mathrm{mg} \mathrm{ml}^{-1}$ in absence of calcium ions, were pipetted onto glowdischarged 200 and 400 mesh gold Quantifoil R1.2/1.3 holey carbon grids (Quantifoil). Grids were blotted for $2-4 \mathrm{~s}$ with a blotting force of 1 at $15^{\circ} \mathrm{C}$ and $100 \%$ humidity and flash-frozen in liquid-ethane using an FEI Vitrobot Mark IV (FEI). Cryo-EM data were collected on a $300 \mathrm{kV}$ FEI Titan Krios electron microscope using a post-column quantum energy filter (Gatan) with a $20 \mathrm{eV}$ slit and a $100 \mu \mathrm{m}$ objective aperture (Extended Data Figs. 1, 2 and 10). Data were collected in an automated fashion using SerialEM ${ }^{36}$ on a K2 Summit detector (Gatan). For the dataset in presence of calcium ions, cryo-EM images were collected at a pixel size of $0.5375 \AA$ in super-resolution mode, a defocus range of -0.5 to $-3.0 \mu \mathrm{m}$, an exposure time of $10 \mathrm{sec}$ and a sub-frame exposure time of $125 \mathrm{~ms}$ (80 frames) with an approximate electron dose of $1 \mathrm{e}^{-} / \AA^{2} /$ frame. To improve the data quality, an extensive effort was made for this dataset to screen different areas within the grid and only regions that provided an estimated resolution of the CTF fit of better than $4 \AA$ were selected for data collection. The dataset in absence of calcium ions was obtained at a pixel size of $0.6825 \AA$ in super-resolution mode, a defocus range of -0.5 to $-3.9 \mu \mathrm{m}$, an exposure time of $15 \mathrm{sec}$ and a sub-frame exposure time of $150 \mathrm{~ms}$ (100 frames) with an electron dose at the specimen level of $0.75-0.8$ $\mathrm{e}^{-} / \AA^{2} /$ frame. The total accumulated dose on the specimen level for both data sets was approximately $80 \mathrm{e}^{-} / \AA^{2}$.

\section{Image processing}


For the dataset collected in presence of calcium ions a total of 4,342 dose-fractionated superresolution images were recorded, 2 x 2 down-sampled by Fourier cropping (final pixel size $1.075 \AA$ ) and subjected to motion correction and dose-weighting of frames by MotionCor $2^{37}$. The contrast transfer function (CTF) parameters were estimated on the movie frames by ctffind $4.1^{38}$. Images showing a strong drift, higher defocus than $-3.0 \mu \mathrm{m}$ or a bad CTF estimation were discarded, resulting in 2,997 images used for further analysis with the software package RELION2.1b1 ${ }^{39}$. Particles were picked automatically using 2D class averages from the previously obtained TMEM16A cryo-EM map as reference ${ }^{14}$ providing an initial set of 629,679 particles. After extraction with a box size of 300 pixels, false positives were eliminated manually or through a first round of 2D classification, resulting in 368,162 particles that were further subjected to several rounds of $2 \mathrm{D}$ classification to remove particles belonging to low-abundance classes. The remaining 252,577 particles were sorted during 3D Classification, a C2 symmetry was imposed and the low-resolution TMEM16A cryo-EM map (EMD-3658) was used as initial model. The best class, comprising 147,368 particles from a total of 2012 images, was subjected to auto-refinement and particle polishing in RELION, with a running average window of 5, a standard deviation of 1 pixel on translations and 200 pixels on particle distance. The final polished and auto-refined map used for model building had a resolution of $4.6 \AA$ before masking and $3.75 \AA$ after masking and was sharpened using an isotropic b-factor of $-106 \AA^{2}$ (Extended Data Figs. 1 and 10). For the data set in absence of calcium ions, a similar workflow for image processing was applied. From a total of 4,738 images (final pixel size $1.365 \AA$ ) $1,349,821$ particles were extracted with a box size of 256 pixels after auto-picking and initial round of $2 \mathrm{D}$ classification. Initial rounds of $2 \mathrm{D}$ and $3 \mathrm{D}$ classification resulted in a set of 467,286 particles, which were subjected to particle polishing and used for final rounds of 3D classification. The final polished and auto-refined map was calculated from 195,465 particles derived from 4726 images with a resolution of $4.86 \AA$ before masking and $4.06 \AA$ after masking and was sharpened using an isotropic b-factor of - 
$116 \AA^{2}$ (Extended Data Figs. 2 and 10). To identify flexible regions both final maps, in presence and absence of calcium ions, were subjected to a final round of 3D Classification. In both cases, three predominant classes were identified that were virtually identical except for the density corresponding to transmembrane $\alpha$-helix 3 and its connecting loops to transmembrane $\alpha$-helix 2 and 4 (Extended Data Figs. 1f and 2d, e). As similar results are obtained for both datasets this region appears to be inherently flexible. The maps had a final resolution of $4.03 \AA, 4.35 \AA$ and $4.19 \AA$ for the classes in presence of calcium ions, and 4.48 $\AA, 4.85 \AA$ and $4.36 \AA$ for the classes in absence of calcium ions. Local resolution estimates were calculated by BlocRes from the Bsoft software package ${ }^{31}$. All resolutions were estimated using the 0.143 cut-off criterion ${ }^{40}$ with gold-standard Fourier shell correlation (FSC) between two independently refined half maps ${ }^{41}$. During post-processing, the approach of highresolution noise substitution was used to correct for convolution effects of real-space masking on the FSC curve ${ }^{42}$.

\section{Model building and Refinement}

The model of the $\mathrm{Ca}^{2+}$-bound form of mTMEM16A was built in $\mathrm{COOT}^{43}$ using the structure of nhTMEM16 (PDBID 4WIS) as template. The electron density was of high quality and allowed interpretation with a model consisting of residues $117-130,165-259,267-466,488-$ 668, 683-910 (Extended Data Figs. 3 and 4). Compared to most parts of the structure, the density of the $\alpha 2-\alpha 3$ loop (residues $441-466$ ), the first part of $\alpha$-helix 3 (residues 488-497) and the $\alpha 3-\alpha 4$ loop (residues 523-534) is not as well defined and the structure may thus be less accurate in these regions. The model was improved by real-space refinement in Phenix ${ }^{44}$, whereby secondary structure elements and the symmetry between both subunits of the dimeric protein were constrained. Reciprocal space refinement was performed with Refmac ${ }^{45,46}$ incorporated in $\mathrm{CCPEM}^{47}$. Coordinates were manually edited in COOT after each refinement 
cycle. The structure of the $\mathrm{Ca}^{2+}$-free from of mTMEM16A was built using the refined $\mathrm{Ca}^{2+}$ bound structure as starting model and by rebuilding regions that have changed its conformation in COOT. Major conformational changes were restricted to the second half of $\alpha$-helix 6. The structure was subsequently refined in Phenix and Refmac as described above. Both models are of high quality (Extended Data Fig. 10a). For validation of the refinement, Fourier shell correlations $\left(\mathrm{FSC}_{\text {sum }}\right)$ between the refined model and the final map were determined (Extended Data Fig. 10b). To monitor the effects of potential over-fitting, random shifts (up to $0.3 \AA$ ) were introduced into the coordinates of the final model, followed by refinement with Refmac against the first unfiltered half-map. The FSC between this shakenrefined model and the first half-map used during validation refinement is termed $\mathrm{FSC}_{\mathrm{work}}$, the FSC against the second half-map, which was not used at any point during refinement $\mathrm{FSC}_{\text {free. }}$. The marginal gap between the curves describing $\mathrm{FSC}_{\text {work }}$ and $\mathrm{FSC}_{\text {free }}$ indicate no over-fitting of the model (Extended Data Fig. 10b).

\section{Poisson-Boltzmann calculations.}

The electrostatic potential was calculated by solving the linearized Poisson-Boltzmann equation in $\mathrm{CHARMM}^{48,49}$ on a $200 \AA$ × $140 \AA$ × $190 \AA$ grid ( $1 \AA$ grid spacing) followed by focusing on a $135 \AA$ x $100 \AA$ x $125 \AA$ grid (0.5 ̊̊ grid spacing). Partial protein charges were derived from the CHARMM36 all-hydrogen atom force field. Hydrogen positions were generated in CHARMM. The protein was assigned a dielectric constant $(\epsilon)$ of 2 . Its transmembrane region was embedded in a 30 - $\AA$-thick slab $(\epsilon=2)$ representing the hydrophobic core of the membrane and two adjacent 15 - $\AA$-thick regions $(\epsilon=30)$ representing the headgroups. This region contained a cylindrical hole around the water-filled intracellular vestibule of one subunit and was surrounded by an aqueous environment $(\epsilon=80)$ containing $150 \mathrm{mM}$ of monovalent mobile ions. 


\section{Electrophysiology.}

The WT mTMEM16A $(a c)$ construct used in this study was described previously ${ }^{14,23}$. The mTMEM16A $(a c)$ cDNA containing only the six extracellular cysteines indispensable for channel function ${ }^{18}(6 \mathrm{C})$, was cloned into the same vector using the FX cloning system ${ }^{50}$. Mutations were introduced using a modified QuikChange method ${ }^{51}$ and were verified by sequencing.

HEK293T cells (ATCC CRL-1573) were transfected at $3 \mu \mathrm{g}$ DNA per $6 \mathrm{~cm}$ petri dish using the calcium phosphate co-precipitation method and were used within 24-96 $\mathrm{h}$ after transfection. Inside-out patches were excised from cells expressing the mTMEM16A construct of interest after the formation of a gigaohm seal. Seal resistance was typically $4-8$ G $\Omega$ or higher. Patch pipettes were pulled from borosilicate glass capillaries with an outer diameter of $1.5 \mathrm{~mm}$ and an inner diameter of $0.86 \mathrm{~mm}$ (Sutter) and were fire-polished using a microforge (Narishige). Pipette resistance was typically 3-8 $\mathrm{M} \Omega$ when filled with recording solutions. Voltage-clamp recordings were performed using Axopatch 200B and Digidata 1550 (Molecular Devices). Analogue signals were filtered at a cut-off frequency of $5 \mathrm{kHz}$ through the in-built 4-pole low-pass Bessel filter of Axopatch 200B and were sampled at $20 \mathrm{kHz}$. Data acquisition was performed using Clampex 10.6 (Molecular Devices). Solution exchange was performed using a theta glass pipette mounted on a high-speed piezo stepper (Siskiyou) for concentration-response experiments. For accessibility experiments, a three-barrelled glass pipette mounted on the SF-77B fast-step stepper (Warner Instruments) was used. The position of the steppers for solution delivery was controlled via analogue voltage signals as programmed in Clampex 10.6. Liquid junction potential was not corrected as this was found to be consistently negligible given the ionic composition of the recording solutions ${ }^{14,23}$. 
Recordings were performed under symmetrical ionic condition. Stock solution with $\mathrm{Ca}^{2+}$ EGTA contains $150 \mathrm{mM} \mathrm{NaCl}, 5.99 \mathrm{mM} \mathrm{Ca}(\mathrm{OH})_{2}, 5 \mathrm{mM}$ EGTA and $10 \mathrm{mM}$ HEPES at $\mathrm{pH}$ 7.40, which corresponds to a free $\mathrm{Ca}^{2+}$ concentration of $1 \mathrm{mM}$. Stock solution with 'EGTA only' contains $150 \mathrm{mM} \mathrm{NaCl}, 5 \mathrm{mM}$ EGTA and $10 \mathrm{mM}$ HEPES at $\mathrm{pH} 7.40$. Free $\mathrm{Ca}^{2+}$ concentrations were adjusted by mixing the stock solutions at the required ratios to yield the corresponding total $\mathrm{Ca}^{2+}$ and EGTA concentrations, which were calculated using the WEBMAXC program (http://web.stanford.edu/ cpatton/webmaxcS.htm).

For concentration-response experiments, current responses at different free $\mathrm{Ca}^{2+}$ concentrations were recorded using a rundown correction protocol ${ }^{23}$. Briefly, a reference concentration of $\mathrm{Ca}^{2+}$ was delivered before and after the test pulse separated by an identical time interval. The response to the test pulse was measured as the ratio of the average of the responses to the pre- and post-pulses. For constructs that do not display noticeable basal activity, background current recorded in the absence of $\mathrm{Ca}^{2+}(5 \mathrm{mM}$ EGTA) was subtracted before analysis. This is not possible for the G644P and the I550A constructs as they display basal activity. The current responses of these mutants were thus analysed without background subtraction. The presence of minimal leak current can be inferred by the strong rectification of the basal current (Extended Data Fig. 9c), which results in inward current with an absolute amplitude of typically less than $30 \mathrm{pA}$ in the dataset analyzed.

To probe the accessibility of the intracellular vestibule we have incubated excised inside-out patches with the small thiol-reactive reagent MTSEA (positively charged) or MTSES (negatively charged) in presence or absence of calcium, and subsequently monitored changes in the current-voltage relationships of activated channels in presence of calcium. MTSEA bromide and sodium MTSES (Anatrace) were dissolved in anhydrous DMSO (Sigma) at $1 \mathrm{M}$, aliquoted and stored under argon at $-20^{\circ} \mathrm{C}$ in a desiccated container. Immediately before the experiment, stock MTS reagents were diluted at the required concentration in the NaCl-based 
recording solutions described above at $1 \mathrm{mM} \mathrm{Ca}^{2+}$ or zero $\mathrm{Ca}^{2+}(5 \mathrm{mM}$ EGTA). MTSEA was used within 15 minutes whereas MTSES, which has a reported half-life of 370 minutes ${ }^{52}$, was used within 2 hours. Modification experiments were performed similar to a described protocol $^{53}$. Specifically, MTS reagents or control solutions without MTS reagents were applied repeatedly to inside-out patches as $2 \mathrm{~s}$ pulses at regular time intervals in the absence or presence of $\mathrm{Ca}^{2+}$. The progress of modification was subsequently monitored at $1 \mathrm{mM} \mathrm{Ca} \mathrm{Ca}^{2+}$ by recording of $\mathrm{I}-\mathrm{V}$ relations using a voltage ramp protocol. The $\mathrm{I}-\mathrm{V}$ relation of the background current recorded in the absence of $\mathrm{Ca}^{2+}$ immediately before the test pulse was subtracted before analysis. In accessibility experiments, we made use of the profound outward rectification of the mutants $\mathrm{K} 588 \mathrm{C}$ and $\mathrm{K} 588 \mathrm{Q} / \mathrm{S} 592 \mathrm{C}$, which disappears upon reaction with MTSEA, as the covalently bound reagent compensates for the lost positive charge in the mutants (Extended Data Fig. 9h, i). Rectification was quantified as the ratio of the absolute current value at $-100 \mathrm{mV}$ to that at $+120 \mathrm{mV}$ (rectification index, RI). Current rundown correction was not required as the readout is already normalized. In the case of MTSES where the absolute magnitude was also measured, the rundown time course was monitored by applying ten pulses of control solution before switching to MTSES-containing solution. Concentration-response data were fitted to a Hill equation. The time course of MTSEA modification was fitted to a sum of two exponentials. Data were analysed using Clampfit 10.6 (Molecular devices), Excel (Microsoft) and Prism 5 (GraphPad) and are presented as mean \pm s.e.m. unless otherwise indicated.

\section{Statistics and Reproducibility}

Electrophysiology data were repeated multiple times from different transfections with very similar results. Conclusions of experiments were not changed upon inclusion of further data. 
In case of constructs not showing basal activity, leaky patches were discarded. In case of mutants showing basal activity, basal currents were strongly rectifying.

\section{Data availability}

The three-dimensional cryo-EM density maps of calcium-bound and calcium-free mTMEM16A have been deposited in the Electron Microscopy Data Bank under accession numbers EMD-3860 and EMD-3861, respectively. The deposition includes corresponding maps calculated with higher b-factors, both half-maps, and the mask used for final FSC calculation. Coordinates for the models of the calcium-bound and calcium-free state have been deposited in the Protein Data Bank under accession numbers 5OYB and 5OYG, respectively. Data can be made available upon reasonable request.

31 Heymann, J. B. \& Belnap, D. M. Bsoft: image processing and molecular modeling for electron microscopy. J. Struct. Biol. 157 (2007).

$32 \mathrm{Yu}, \mathrm{K}$. et al. Identification of a lipid scrambling domain in ANO6/TMEM16F. eLife 4, e06901, doi:10.7554/eLife.06901 (2015).

33 Smart, O. S., Neduvelil, J. G., Wang, X., Wallace, B. A. \& Sansom, M. S. HOLE: a program for the analysis of the pore dimensions of ion channel structural models. $J$. Mol. Graph. 14, 354-360, 376 (1996).

34 Geertsma, E. R., Nik Mahmood, N. A., Schuurman-Wolters, G. K. \& Poolman, B. Membrane reconstitution of $\mathrm{ABC}$ transporters and assays of translocator function. Nat. Protoc. 3, 256-266 (2008).

35 Kane Dickson, V., Pedi, L. \& Long, S. B. Structure and insights into the function of a $\mathrm{Ca}(2+)$-activated $\mathrm{Cl}(-)$ channel. Nature 516, 213-218 (2014). 
36 Mastronarde, D. N. Automated electron microscope tomography using robust prediction of specimen movements. J. Struct. Biol. 152, 36-51 (2005).

37 Zheng, S., Palovcak, E., Armache, J.-P., Cheng, Y. \& Agard, D. Anisotropic Correction of Beam-induced Motion for Improved Single-particle Electron Cryomicroscopy. bioRxiv, doi:10.1101/061960 (2016).

38 Rohou, A. \& Grigorieff, N. CTFFIND4: Fast and accurate defocus estimation from electron micrographs. J. Struct. Biol. 192, 216-221 (2015).

39 Kimanius, D., Forsberg, B. O., Scheres, S. H. \& Lindahl, E. Accelerated cryo-EM structure determination with parallelisation using GPUs in RELION-2. elife 5, doi:10.7554/eLife.18722 (2016).

40 Rosenthal, P. B. \& Henderson, R. Optimal determination of particle orientation, absolute hand, and contrast loss in single-particle electron cryomicroscopy. J. Mol. Biol. 333, 721-745 (2003).

41 Scheres, S. H. \& Chen, S. Prevention of overfitting in cryo-EM structure determination. Nat. Methods 9, 853-854 (2012).

42 Chen, S. et al. High-resolution noise substitution to measure overfitting and validate resolution in 3D structure determination by single particle electron cryomicroscopy. Ultramicroscopy 135, 24-35 (2013).

43 Emsley, P. \& Cowtan, K. Coot: model-building tools for molecular graphics. Acta Crystallogr. D Biol. Crystallogr. 60, 2126-2132 (2004).

44 Adams, P. D. et al. PHENIX: building new software for automated crystallographic structure determination. Acta Crystallogr. D Biol. Crystallogr. 58, 1948-1954 (2002).

45 Murshudov, G. N. et al. REFMAC5 for the refinement of macromolecular crystal structures. Acta Crystallogr. D Biol. Crystallogr. 67, 355-367 (2011). 
46 Brown, A. et al. Tools for macromolecular model building and refinement into electron cryo-microscopy reconstructions. Acta Crystallogr. D Biol. Crystallogr. 71, $136-153(2015)$.

47 Wood, C. et al. Collaborative computational project for electron cryo-microscopy. Acta Crystallogr. D Biol. Crystallogr. 71, 123-126 (2015).

48 Brooks, B. R. et al. CHARMM: a program for macromolecular energy, minimization, and dynamics calculations. J. Comp. Chem. 4, 187-217 (1983).

49 Im, W., Beglov, D. \& Roux, B. Continuum solvation model: Electrostatic forces from numerical solutions to the Poisson-Bolztmann equation. Comput. Phys. Commun. 111, 59-75 (1998).

50 Geertsma, E. R. \& Dutzler, R. A versatile and efficient high-throughput cloning tool for structural biology. Biochemistry 50, 3272-3278 (2011).

51 Zheng, L., Baumann, U. \& Reymond, J. L. An efficient one-step site-directed and sitesaturation mutagenesis protocol. Nucleic Acids Res. 32, e115, doi:10.1093/nar/gnh110 (2004).

52 Karlin, A. \& Akabas, M. H. Substituted-cysteine accessibility method. Methods Enzymol. 293, 123-145 (1998).

53 Contreras, J. E. et al. Voltage profile along the permeation pathway of an open channel. Biophys. J. 99, 2863-2869 (2010).

\section{Extended Data Figure 1 | Functional characterization and cryo-EM reconstruction of} mTMEM16A in a $\mathrm{Ca}^{2+}$-bound form. a, Left, $\mathrm{Ca}^{2+}$ concentration-response relationships measured from patches excised from a stable cell line expressing mTMEM16A. Data were recorded at -80 and $80 \mathrm{mV}$ and show mean values of normalized currents of 5 biological 
replicates, errors are s.e.m. Solid lines show a fit to a Hill equation. A representative current trace is shown on the right. Numbers above the current traces indicate the $\mathrm{Ca}^{2+}$ concentration $(\mu \mathrm{M})$, blue and violet dots the reference pulses used for rundown correction at +80 and -80 $\mathrm{mV}$ respectively. b, Fluorescence-based $\mathrm{Cl}^{-}$flux assay into proteoliposomes containing mTMEM16A in the presence (green) and absence of $\mathrm{Ca}^{2+}$ (red). Transport into protein-free liposomes is shown for comparison (blue). $\mathrm{H}^{+}$transport into proteoliposomes accompanying concentration-gradient driven influx of $\mathrm{Cl}^{-}$is monitored by the decrease of the fluorescence of 9-amino-6-chloro-2-methoxyacridine (ACMA). Time zero corresponds to addition of the $\mathrm{H}^{+}$ ionophore CCCP. Experiments show mean of 4 technical replicates, errors are s.e.m. c, Representative cryo-EM image and $\mathbf{d}, 2 \mathrm{D}$ class averages of vitrified mTMEM16A in a $\mathrm{Ca}^{2+}$ bound state. e, Angular distribution plot of particles included in the final C2-symmetric 3D reconstruction. The number of particles with respective orientations are represented by length and color of the cylinders. f, 3D Classification of the final map performed to identify structural variances. Predominant classes are shown as maps with differences highlighted in red. The fraction of total particles in each class $(\%)$ is indicated, the final resolution after refinement and masking is shown where applicable. 3D classification revealed three predominant classes. Structural variations, between these marginally different populations, concern $\alpha$-helix 3 , which is generally less well defined than the other transmembrane segments, and the connecting $\alpha 2-\alpha 3$ and $\alpha 3-\alpha 4$ loops. g, Final reconstruction map colored by local resolution as calculated by BlocRes from the Bsoft software package ${ }^{31} . \mathbf{h}$, Fourier shell correlation (FSC) plot of the final refined unmasked (red) and masked (green) mTMEM16A map in presence of calcium ions. The resolution at which the curve drops below the 0.143 threshold is indicated. A thumbnail of the mask used for FSC calculation overlaid on the atomic model is shown on the upper right corner. 


\section{Extended Data Figure 2 | Cryo-EM reconstruction of mTMEM16A in a $\mathrm{Ca}^{2+}$-free form.}

a, Representative cryo-EM image and $\mathbf{b}, 2 \mathrm{D}$ class averages of vitrified mTMEM16A in a $\mathrm{Ca}^{2+}$-free state. c, Angular distribution plot of particles included in the final C2-symmetric 3D reconstruction. The number of particles with respective orientations are represented by length and color of the cylinders. d, 3D Classification of the final map performed to identify structural variances. Predominant classes are shown as maps with differences highlighted in red. The fraction of total particles in each class $(\%)$ is indicated, the final resolution after refinement and masking is shown where applicable. As for the $\mathrm{Ca}^{2+}{ }_{-}$bound structure 3D classification revealed three predominant classes with pronounced variation in the less well defined $\alpha$-helix 3 and the connecting $\alpha 2-\alpha 3$ and $\alpha 3-\alpha 4$ loops. e, Superposition of EM density at the extracellular part of $\alpha 3$ of two major classes. f, Final reconstruction map colored by local resolution as calculated by BlocRes from the Bsoft software package ${ }^{31} \cdot \mathbf{g}$, Fourier shell correlation (FSC) plot of the final refined unmasked (red) and masked (violet) mTMEM16A in absence of calcium ions. The resolution at which the curve drops below the 0.143 threshold is indicated. A thumbnail of the mask used for FSC calculation overlaid on the atomic model is shown on the upper right corner. $\mathbf{h}$, Projections of $\mathrm{Ca}^{2+}$-free and $\mathrm{Ca}^{2+}$-bound conformations of mTMEM16A orthogonal to the membrane. The movement of $\alpha$-helix 6 has perturbed the detergent micelle in the $\mathrm{Ca}^{2+}$-free structure, which has become partially discontinuous near the intracellular vestibule.

Extended Data Figure 3 | Cryo-EM density. a, Sections of the cryo-EM density of the $\mathrm{Ca}^{2+}$ bound (grey, contoured at $5.5 \sigma$ ) and $\mathrm{Ca}^{2+}$-free (blue, contoured at $4.5 \sigma$ ) densities superimposed on part of the respective refined structures. Structures are shown as sticks in unique colors, structural elements and data sets $\left(\mathrm{Ca}^{2+}\right.$, no $\left.\mathrm{Ca}^{2+}\right)$ are labelled. b, Electron 
density (grey, contoured at $4.5 \sigma$ ) of the $\mathrm{Ca}^{2+}$-bound protein around disulfide bonds in the extracellular domain superimposed on the refined model.

Extended Data Figure 4 | Cryo-EM density of selected regions. a, Stereo view of cryo-EM density $(3.5 \sigma)$ around the $\alpha 2-\alpha 3$ and the $\alpha 4-\alpha 5$ loop of the $\mathrm{Ca}^{2+}$-bound structure shown superimposed on the refined model. b, Stereo view of the occupied $\mathrm{Ca}^{2+}$-binding site with cryo-EM density (5.5 $\sigma$ ) shown superimposed on the refined model of the $\mathrm{Ca}^{2+}$-bound state. c, Stereo view of the vacant $\mathrm{Ca}^{2+}$ binding region in the $\mathrm{Ca}^{2+}$-free structure of mTMEM16A with cryo-EM density (4.5 $\sigma$ ) shown superimposed on the refined model. a-c, Selected $\alpha$-helices are labeled.

Extended Data Figure 5 | mTMEM16A sequence and structural features. a, Sequence of the mTMEM16A $(a c)$ isoform (UniProt Q8BHY3.2) used in this study. Secondary structure elements are shown below (transmembrane domain in green, $\mathrm{N}$-terminal domain in violet, $\mathrm{C}$ terminal region in grey, $\alpha 1-\alpha 2$ loop in blue, $\alpha 2-\alpha 3$ loop in black, $\alpha 5-\alpha 6$ loop in beige and $\alpha 9$ $\alpha 10$ loop in red). $\boldsymbol{\nabla}$ indicates the position of cysteines involved in disulfide bonds, $\bullet$, glycines of $\alpha 6$ that serve as hinges in conformational transitions and, $\boldsymbol{\Delta}$, residues of the $\mathrm{Ca}^{2+}$-binding site. The sequence inserted in $c$ isoforms is highlighted in yellow, the preceding stretch of glutamates in red. b, Ribbon representation of the mTMEM16A subunit. The colouring is as in a, disulfide bridges are indicated in yellow. c, Sequence alignment of a section of $\alpha 6$ in different family members. Shown are the human orthologues, mTMEM16 and fungal family members. Selected residues are highlighted. Insertion in TMEM16A and B is indicated by an asterisk. d, Dimer interface. Shown is a $\mathrm{C} \alpha$-representation of the symmetry related extracellular part of $\alpha$-helix 10. Interacting residues are labelled and their side-chains are 
displayed as sticks. The interface is predominantly hydrophobic but contains a pair of $\mathrm{H}$ bonds between Gln 872 and Asn 873 at the inner end of the contact region located at the centre of the membrane, equivalent to interactions between conserved glutamate and histidine residues formed in TMEM16 scramblases $^{13}$. e, Ribbon representation of the extracellular domain. Extracellular parts of transmembrane helices are coloured in green. The loops are shown in unique colours $(\alpha 1-\alpha 2$, in blue, $\alpha 5-\alpha 6$ in beige, $\alpha 9-\alpha 10$ in red). The extracellular domain is stabilized by at least three disulfide bridges (indicated in yellow), two within the $\alpha 1-\alpha 2$ loop and another connecting the $\alpha 1-\alpha 2$ with the $\alpha 9-\alpha 10$ loop, which likely explains why mutation of any of these six cysteine residues causes a loss of function of the channel ${ }^{18}$. A fourth disulfide bridge is potentially formed between two adjacent cysteines in the $\alpha 5-\alpha 6$ loop. $\mathrm{d}$, e, The view is rotated by $90^{\circ}$ around the dimer axis compared to Fig. 1. f, N-terminal domain. Ribbon representation of the N-terminal domain of mTMEM16A is shown on top, a $\mathrm{C} \alpha$-representation of a superposition of the equivalent domains of mTMEM16A (green) and nhTMEM16 (red) at the bottom. g, Ribbon representation of the interacting $\alpha 2-\alpha 3$ (black) and $\alpha 4-\alpha 5$ (green) loops. The sequence corresponding to the $c$ segment (EAVK) of mTMEM16 splice variants is coloured in yellow, the preceding stretch of glutamates (EEEE) in red. The proximity of the $\alpha 2-\alpha 3$ loop to the short $\alpha 4-\alpha 5$ loop, the latter of which having been previously proposed to carry residues that distinguish TMEM16 channels from scramblases ${ }^{32}$, suggest a potential coupling to the transmembrane domain. Although the $\alpha 2-\alpha 3$ loop is not directly involved in high affinity $\mathrm{Ca}^{2+}$ binding leading to channel activation, a stretch of glutamates adjacent to the $c$ region might still interact with $\mathrm{Ca}^{2+}$ and thus be responsible for the observed increase in the open probability at high ligand concentration ${ }^{23}$. e-g, Selected secondary structure elements are labelled. 
Extended Data Figure 6 | Superposition of TMEM16 structures. a, Stereo view of a superposition of the $\mathrm{Ca}^{2+}$-bound (green) and $\mathrm{Ca}^{2+}$-free (violet) structures of the mTMEM16A ion channel. The proteins are displayed as $\mathrm{C} \alpha$ representations. $\mathbf{b}$, Root mean square deviations (RMSDs) of $\mathrm{C} \alpha$ atoms in the superposition shown in a. The resemblance between the ligandbound and ligand-free structures is reflected in the low RMSD of $0.5 \AA$ for $692 \mathrm{C} \alpha$ positions encompassing the entire subunit except for the inner half of $\alpha$-helix 6 . In contrast to the bulk of the protein, the RMSD of $6.5 \AA$ for $17 \mathrm{C} \alpha$ atoms of the inner part of $\alpha$-helix 6 indicates a large conformational change upon binding of $\mathrm{Ca}^{2+}$. Apart from the specified movements, no obvious distortions in the structure of the $\mathrm{Ca}^{2+}$-bound conformation are observed compared to the protein that was never exposed to $\mathrm{Ca}^{2+}$ during purification. This is despite the fact that in patch-clamp experiments mTMEM16A becomes inactive upon a prolonged exposure to the ligand ${ }^{28}$. c, Stereo view of a superposition of the $\mathrm{Ca}^{2+}$-bound structures of the mTMEM16A ion channel (green) and the nhTMEM16 lipid scramblase (brown). Proteins are shown as ribbons. d, RMSDs of $\mathrm{C} \alpha$ atoms from a superposition of conserved secondary structure elements of mTMEM16A on nhTMEM16 shown in c. The general resemblance of the mTMEM16A structure to the structure of the lipid scramblase nhTMEM16 is illustrated by the RMSD of $4.0 \AA$ between $447 \mathrm{C} \alpha$ positions. a, c, Blue spheres correspond to bound $\mathrm{Ca}^{2+}$ ions. $\mathrm{b}, \mathrm{d}$, Individual positions are displayed (x). Transmembrane helices are indicated below for mTMEM16A (b) and nhTMEM16 (d), respectively.

Extended Data Figure 7 | Pore geometry. a, Ion conduction pore of mTMEM16A in the $\mathrm{Ca}^{2+}$-bound conformation. The accessible surface of the pore (generated with a solvent probe of diameter $2.2 \AA$ ) is shown as grey mesh, bound $\mathrm{Ca}^{2+}$ as blue spheres. $\mathbf{b}$, Pore diameter along the pore-axis of mTMEM16A in the $\mathrm{Ca}^{2+}$-bound conformation as calculated with the program $\mathrm{HOLE}^{33}$. c, View of interactions between the extracellular parts of $\alpha$-helices 4 and 6 in the 
$\mathrm{Ca}^{2+}$-bound structure. $\mathbf{d}$, Ion conduction pore of mTMEM16A in the $\mathrm{Ca}^{2+}$-free conformation. The accessible surface was generated as in a. e, Pore diameter along the pore-axis of mTMEM16A in the $\mathrm{Ca}^{2+}$-free conformation (violet) compared to diameter of the $\mathrm{Ca}^{2+}$-bound pore (green dashed line). $\mathbf{f}$, Difference in the pore diameter between $\mathrm{Ca}^{2+}$-free and $\mathrm{Ca}^{2+}$-bound conformations. Negative values reflect the tighter geometry of the pore in the $\mathrm{Ca}^{2+}$-free conformation. g, Stereo view of a superposition of the pore regions in the $\mathrm{Ca}^{2+}$-bound (green) and $\mathrm{Ca}^{2+}$-free (violet) conformations of mTMEM16A. a, c ,e, g, $\alpha$-helices constituting the ion conduction pore are shown in $\mathrm{C} \alpha$-representation, side-chains of pore-lining residues as sticks. a, b, d-f: $\bullet, \boldsymbol{\Lambda}, \boldsymbol{\nabla}$, indicate equivalent locations in the pore.

Extended Data Figure 8 | Pore electrostatics and $\mathrm{Ca}^{2+}$-binding site mutants. a, Structure of mTMEM16A in the $\mathrm{Ca}^{2+}$-bound state used for calculation of the electrostatic potential. Positions along which the pore potential was plotted are shown as orange spheres. A close-up of the $\mathrm{Ca}^{2+}$-binding site is shown on the right. Bound $\mathrm{Ca}^{2+}$ ions are displayed as blue spheres. b, Electrostatic potential along the pore of mTMEM16A in the $\mathrm{Ca}^{2+}$-bound conformation as determined by a numerical solution of the Poisson-Boltzmann equation. c, Structure of the $\mathrm{Ca}^{2+}$-free state used for calculation of the electrostatic potential in a similar representation as in a. Close-up of the empty ligand binding-site is shown on the right. d, Electrostatic potential along the pore of mTMEM16A in the $\mathrm{Ca}^{2+}$-free conformation determined as in $\mathrm{b}$ (violet) compared to the potential of the $\mathrm{Ca}^{2+}$-bound state (green dashed line). a, c, The protein is shown as ribbon, the view is from within the membrane on one of the two pores in the dimeric protein. The membrane boundary is indicated by black lines with the hydrophobic core in the centre and the two headgroup regions above and below. Violet spheres in the close-ups correspond to the position at which the electrostatic potential in the $\mathrm{Ca}^{2+}$-free conformation is at its minimum. $*, \boldsymbol{\Delta}, \boldsymbol{\nabla}$, indicate equivalent locations in the pore. $\mathrm{Ca}^{2+}$ concentration- 
response relationships (left) and representative current traces (right) of mTMEM16A, e, WT and mutants, f, N651A, g, N730A. e-g Data on the left panels were measured from excised patches at -80 and $80 \mathrm{mV}$ and show mean values of normalized currents of 5 (WT, N651A) and 7 (N730A) biological replicates, errors are s.e.m. Solid lines show a fit to a Hill equation. Dashed lines in $\mathrm{f}, \mathrm{g}$, indicate the relations of WT at the same voltages. Numbers above the current traces indicate the $\mathrm{Ca}^{2+}$ concentration $(\mu \mathrm{M})$, blue and violet dots the reference pulses used for rundown correction at +80 and $-80 \mathrm{mV}$ respectively.

Extended Data Figure 9 | Electrophysiology. $\mathrm{Ca}^{2+}$ concentration-response relationships (left) and representative current traces (right) of mTMEM16A mutants a, G644A, b, G644P. c, Rectification of the basal current of G644P. Left, representative current recorded at $80\left(\mathrm{I}_{80}\right)$ and $-80 \mathrm{mV}(\mathrm{I}-80)$ at 0 (blue) and $2 \mu \mathrm{M} \mathrm{Ca}^{2+}$ (orange). Right, rectification index (defined as $\left.\mathrm{I}_{80} /-\mathrm{I}_{-80}\right)$ at 0 and $2 \mu \mathrm{M} \mathrm{Ca}^{2+}$. Values and mean of 7 biological replicates are displayed, errors are s.e.m. $\mathrm{Ca}^{2+}$ concentration-response relationships (left) and representative current traces (right) of the mTMEM16A mutants d, G656A, e, G656P, f, P658A, g, P658G. h, Left, time dependence of modification of a mutant of mTMEM16A only containing 6 essential cysteines $^{18}$ (6C) with $5 \mathrm{mM}$ MTSEA in the presence of $1 \mathrm{mM} \mathrm{Ca}^{2+}$ monitored by the change of the rectification index $\left(-\mathrm{I}_{-100} / \mathrm{I}_{120}\right)$. Right, time dependence of the modification of $6 \mathrm{C}$ K588C by $2.5 \mathrm{mM}$ MTSEA in the presence of $1 \mathrm{mM} \mathrm{Ca}^{2+}$. Experiments with $5 \mathrm{mM}$ of the positively charged MTSEA are shown as dashed lines for comparison. Data show the mean of $5\left(6 \mathrm{C} \mathrm{Ca}^{2+}\right)$ or 7 (6C-K588C Ca ${ }^{2+}, 2.5 \mathrm{mM}$ MTSEA) biological replicates, errors are s.e.m. Dashed line corresponds to data shown in Fig. 4f. i, Normalized I-V relationships obtained using a ramp protocol prior to (beige) and after (blue) application of MTSEA to different constructs in the presence of $1 \mathrm{mM} \mathrm{Ca}^{2+}$ (unless stated otherwise). From left to right, $6 \mathrm{C}$, the mutant $6 \mathrm{C}-\mathrm{K} 588 \mathrm{C}$ constructed on the same background, the mutant 6C-K588C with MTSEA 
applied in the absence of $\mathrm{Ca}^{2+}$, the mutant 6C-K588Q/S592C. $\mathbf{j}$, Normalized I-V relationships obtained using a ramp protocol prior to (grey) and after (blue) application of the negatively charged MTSES in the presence of $1 \mathrm{mM} \mathrm{Ca}^{2+}$ for WT (left) and S592C (right). i, j, Shown are the averages of $5\left(6 \mathrm{C} \mathrm{Ca}^{2+}\right), 7\left(6 \mathrm{C}-\mathrm{K} 588 \mathrm{C} \mathrm{Ca}^{2+}\right), 8\left(6 \mathrm{C}-\mathrm{K} 588 \mathrm{C}\right.$ no $\left.\mathrm{Ca}^{2+}\right), 4(6 \mathrm{C}-\mathrm{S} 592 \mathrm{C}$ $\left.\mathrm{Ca}^{2+}\right), 4\left(\right.$ WT $\left.\mathrm{Ca}^{2+}\right)$ and $5\left(\mathrm{~S} 592 \mathrm{C} \quad \mathrm{Ca}^{2+}\right)$ biological replicates. Time dependence of modification of WT (left) and S592C (right) with $10 \mathrm{mM}$ MTSES. k, Time-dependent changes of the rectification index after incubation with 10 mM MTSES. Data show the mean of $6\left(\mathrm{WT} \mathrm{Ca}^{2+}\right)$ or $5\left(\mathrm{~S} 592 \mathrm{C} \mathrm{Ca}^{2+}\right)$ biological replicates, errors are s.e.m. 1, Time-dependent changes of the current amplitude at $80 \mathrm{mV}$ for the WT (left) and S592C (right) after incubation with $10 \mathrm{mM}$ MTSES. The decay of the current due to rundown is indicated as dashed line. Data show the mean of $9\left(\mathrm{WT} \mathrm{Ca}^{2+}\right)$ or $7\left(\mathrm{~S} 592 \mathrm{C} \mathrm{Ca}^{2+}\right)$ biological replicates, errors are s.e.m. $\mathbf{m}, \mathrm{Ca}^{2+}$ concentration-response relationships (top) and representative current traces (bottom) of the mTMEM16A mutant I550A. a, b, d-g, m, Data on the left panels were measured from excised patches at -80 and $80 \mathrm{mV}$ and show mean values of normalized currents of 6 (G644A, P658A), 7 (G644P, G656P, I550A) or 8 (G656A, P658G) biological replicates, errors are s.e.m. Solid lines show a fit to a Hill equation. Data recorded at $80 \mathrm{mV}$ and $-80 \mathrm{mV}$ are shown in blue and violet respectively. Dashed lines indicate the relations of WT at the same voltages (Extended Data Fig. 8e). Numbers above the current traces indicate the $\mathrm{Ca}^{2+}$ concentration $(\mu \mathrm{M})$, blue and violet dots indicate the reference pulses used for rundown correction at +80 and $-80 \mathrm{mV}$ respectively.

Extended Data Figure 10 | Statistics and validation. a, Statistics of cryo-EM data collection, 3D reconstruction and model refinement. Fourier shell correlation curves of refined models versus maps of mTMEM16a in presence, $\mathbf{b}$, and absence, $\mathbf{c}$, of calcium ions for cross-validation. The green (b) and violet (c) curve show the FSC curve for the refined 
model compared to the full masked dataset $\left(\mathrm{FSC}_{\text {sum }}\right)$. The FSC curve for the refined model compared to the masked half-map 1 ( $\mathrm{FSC}_{\text {work}}$, used during validation refinement) is shown in blue and for the refined model compared to the masked half-map 2 ( $\mathrm{FSC}_{\text {free}}$, not used during validation refinement) in grey. Dashed lines indicate the FSC threshold used for $\mathrm{FSC}_{\text {sum }}$ of 0.5 and for $\mathrm{FSC}_{\text {free/work }}$ of 0.143 . 

b extracellular vestibule

a

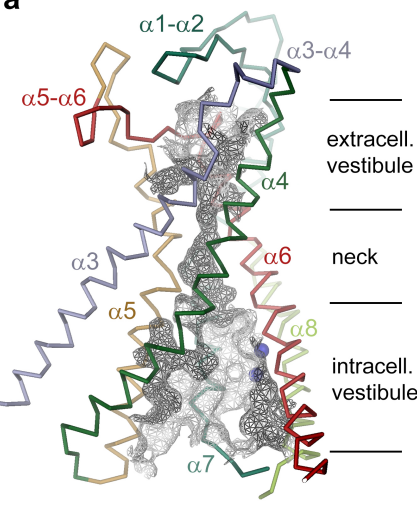

C

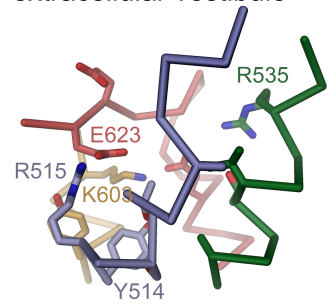

d intracellular vestibule

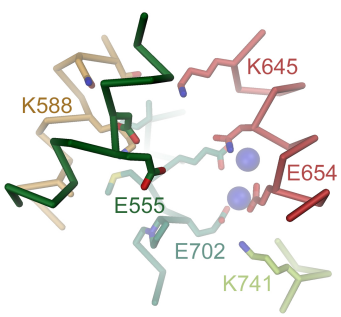

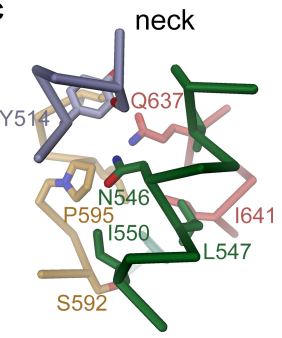

e

$\mathrm{Ca}^{2+}$-binding site

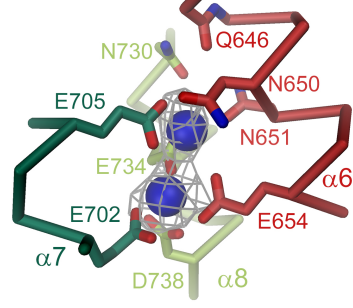


a

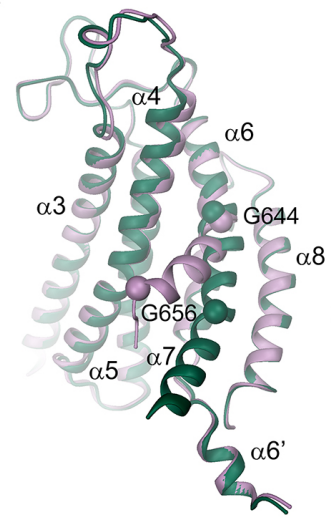

b

C

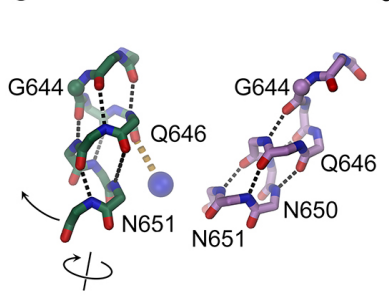

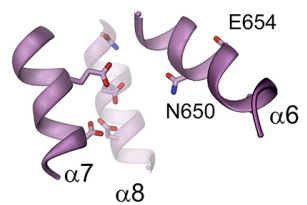

d

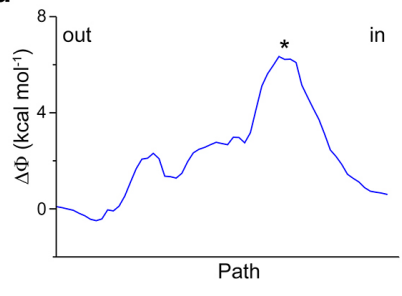


b

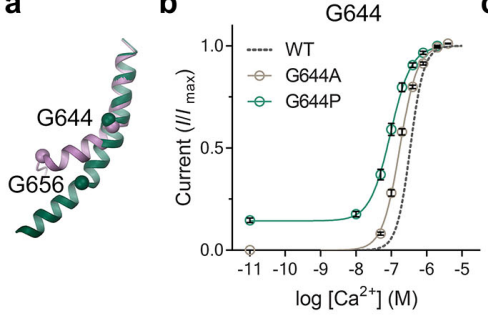

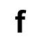

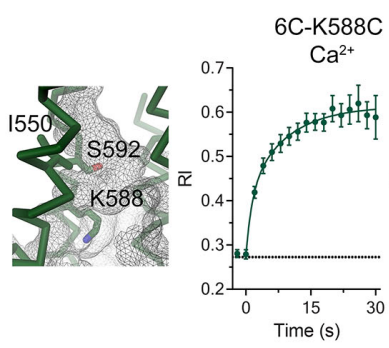

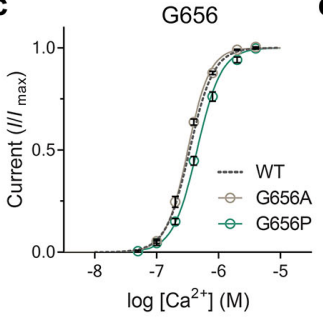

h $\begin{array}{ll}\text { d } & \mathrm{P} 658\end{array}$

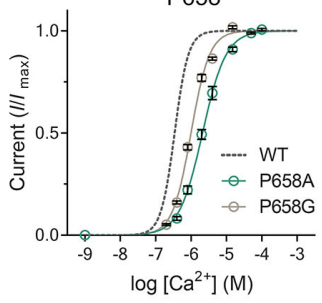

g
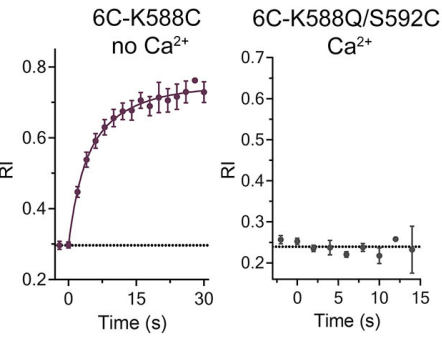

I550A
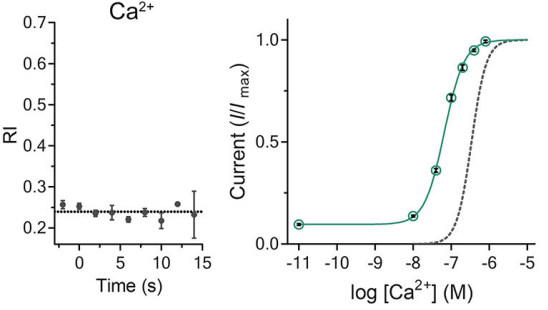
$a$

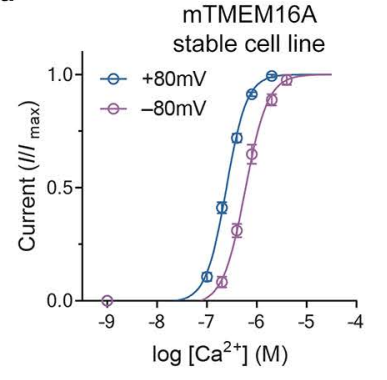

c

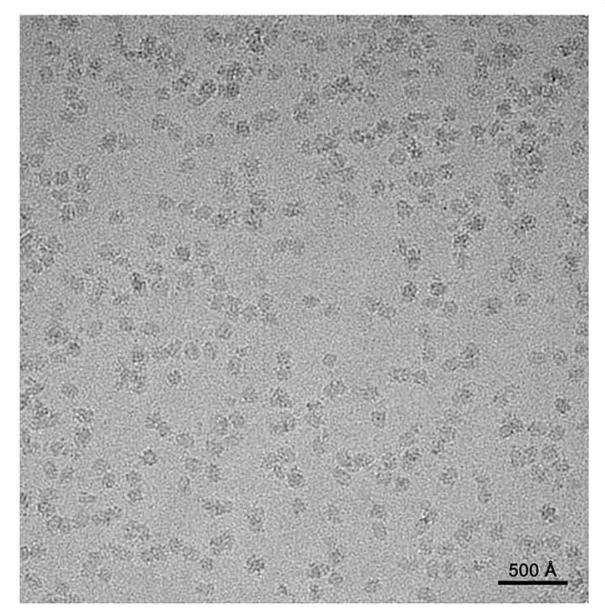

Initial Selection

4,392 micrographs 629,679 autopicked particles d

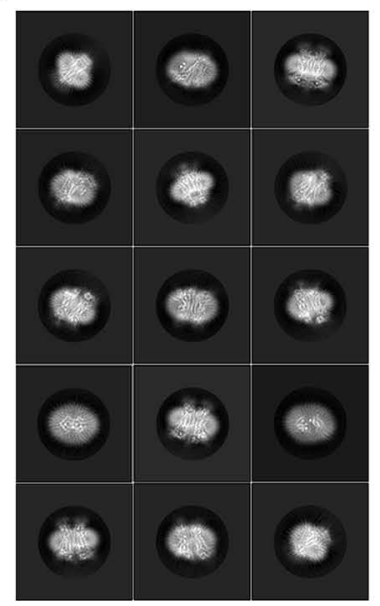

mTMEM16A

reconstitution

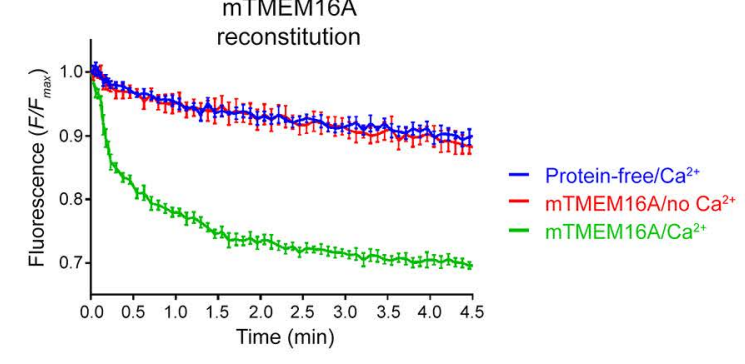

e
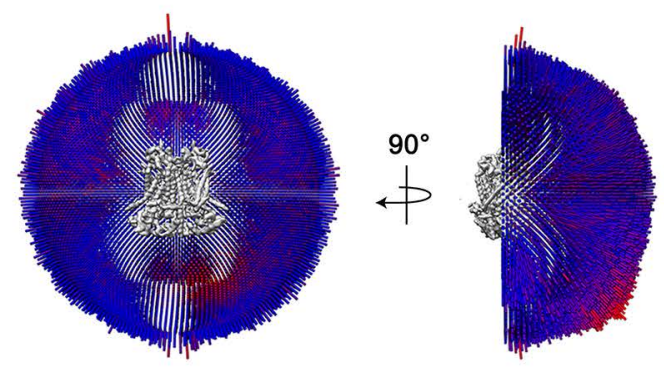

Final Map

2,012 micrographs 147,383 particles

$3.75 \AA$

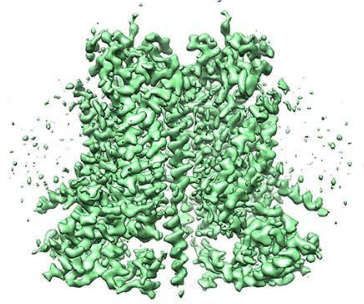

3D Classification

\begin{tabular}{cccccc}
\hline & & & & & \\
Class1 & Class 2 & Class3 & Class 4 & Class3 & Class 6 \\
$24 \%$ & $13.2 \%$ & $18.9 \%$ & $11.1 \%$ & $22.7 \%$ & $9.5 \%$ \\
$4.03 \AA$ & & $4.35 \AA$ & & $4.19 \AA$ &
\end{tabular}
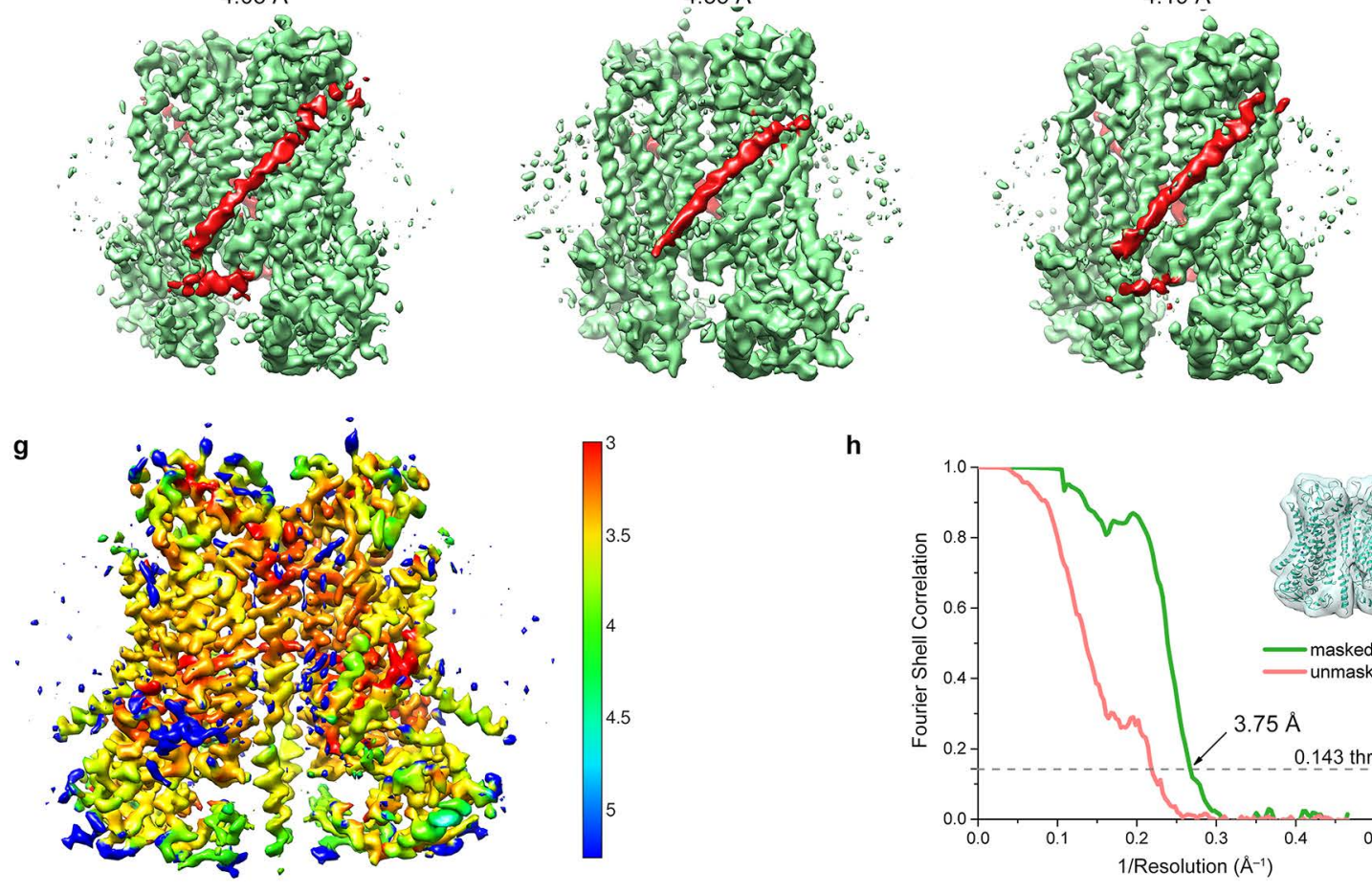

h

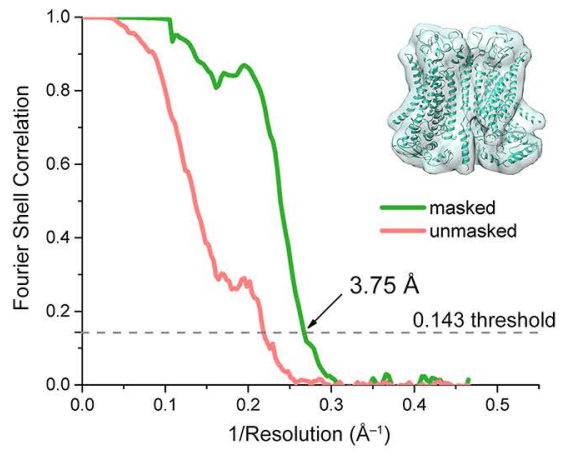


a

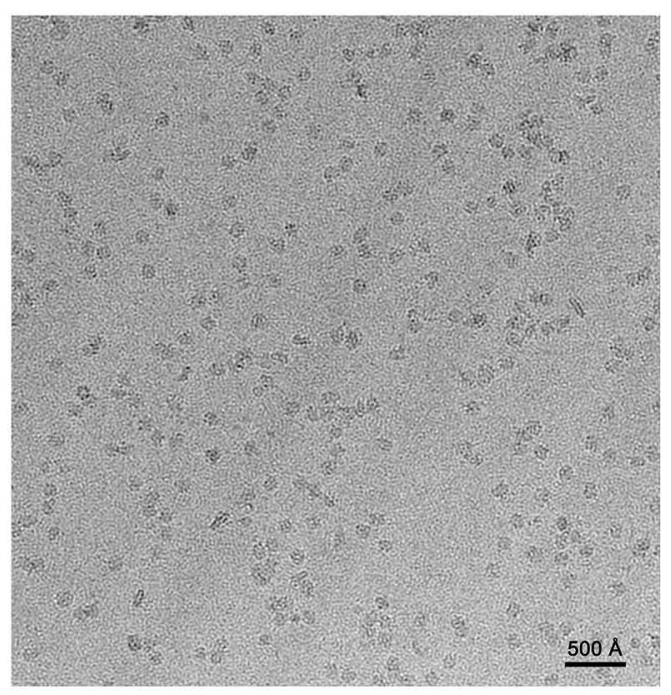

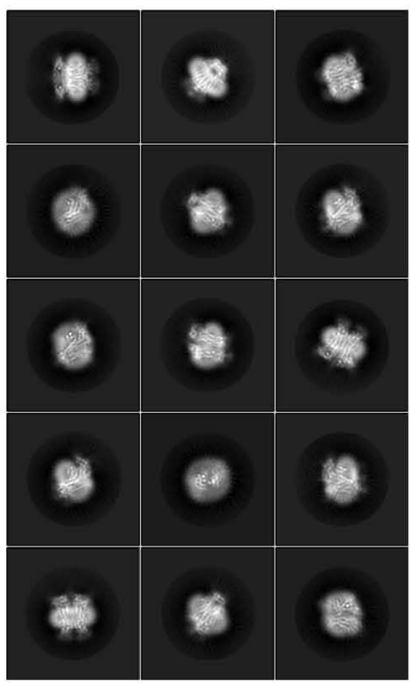

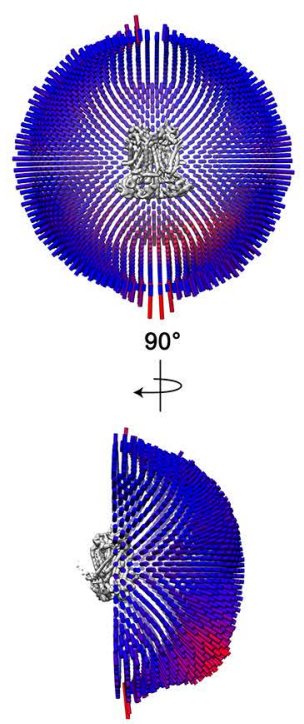

e

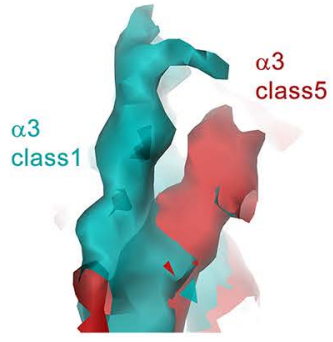

3D Classification

Class 1

$24 \%$

$4.48 \AA$

Initial Selection

5,063 micropgraphs $1,349,821$ particles
Final Map

4,726 micropgraphs

195,465 particles

$4.06 \AA$
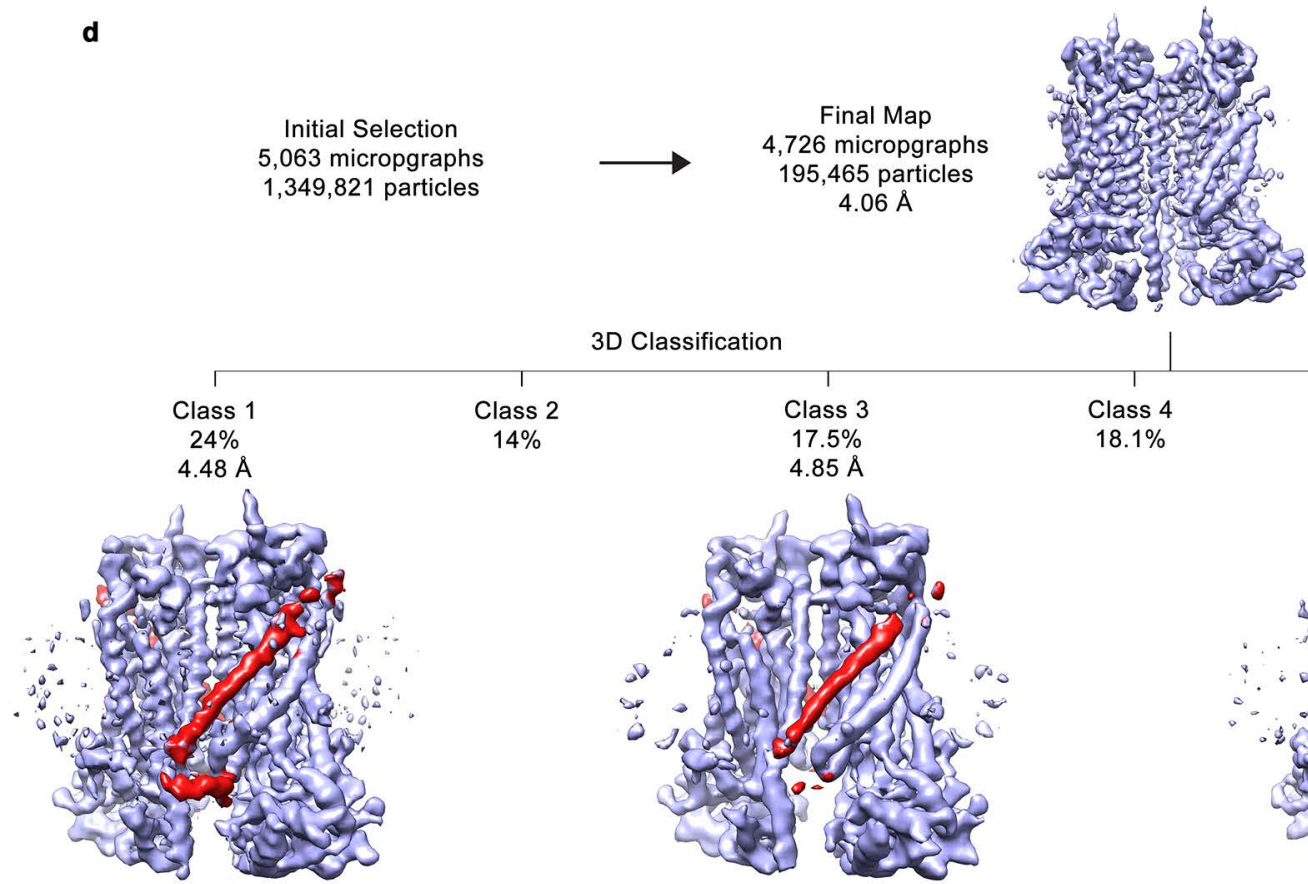

\section{Class 2}

$14 \%$

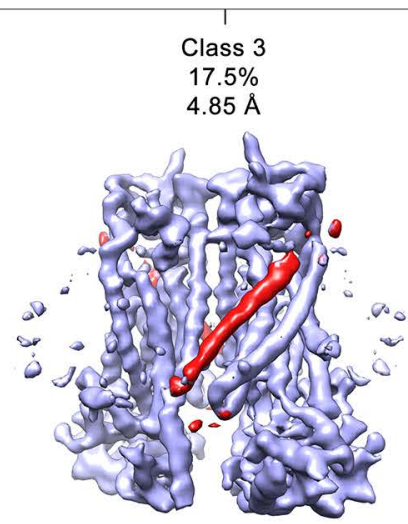

Class 4
$18.1 \%$

Class 5

$25.7 \%$

$4.36 \AA$

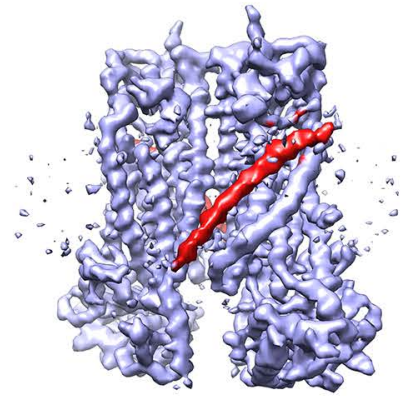

h

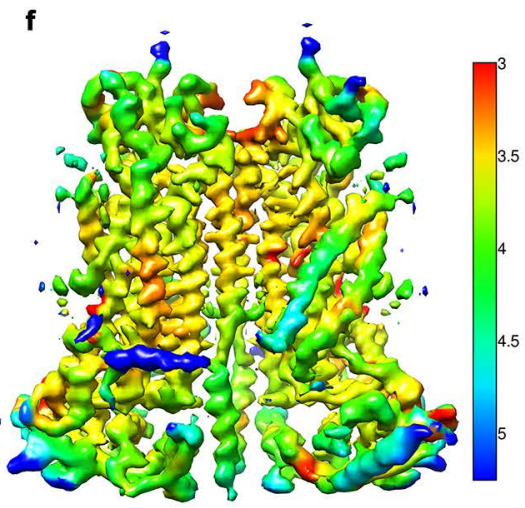

g

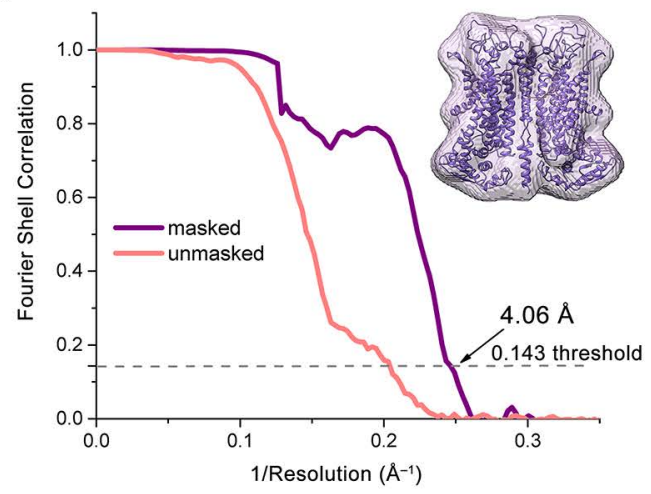

mTMEM16A noCa ${ }^{2+}$

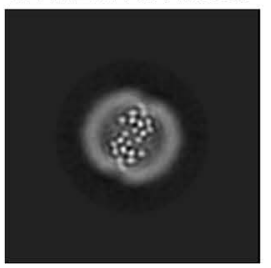

mTMEM16A Ca ${ }^{2+}$ 

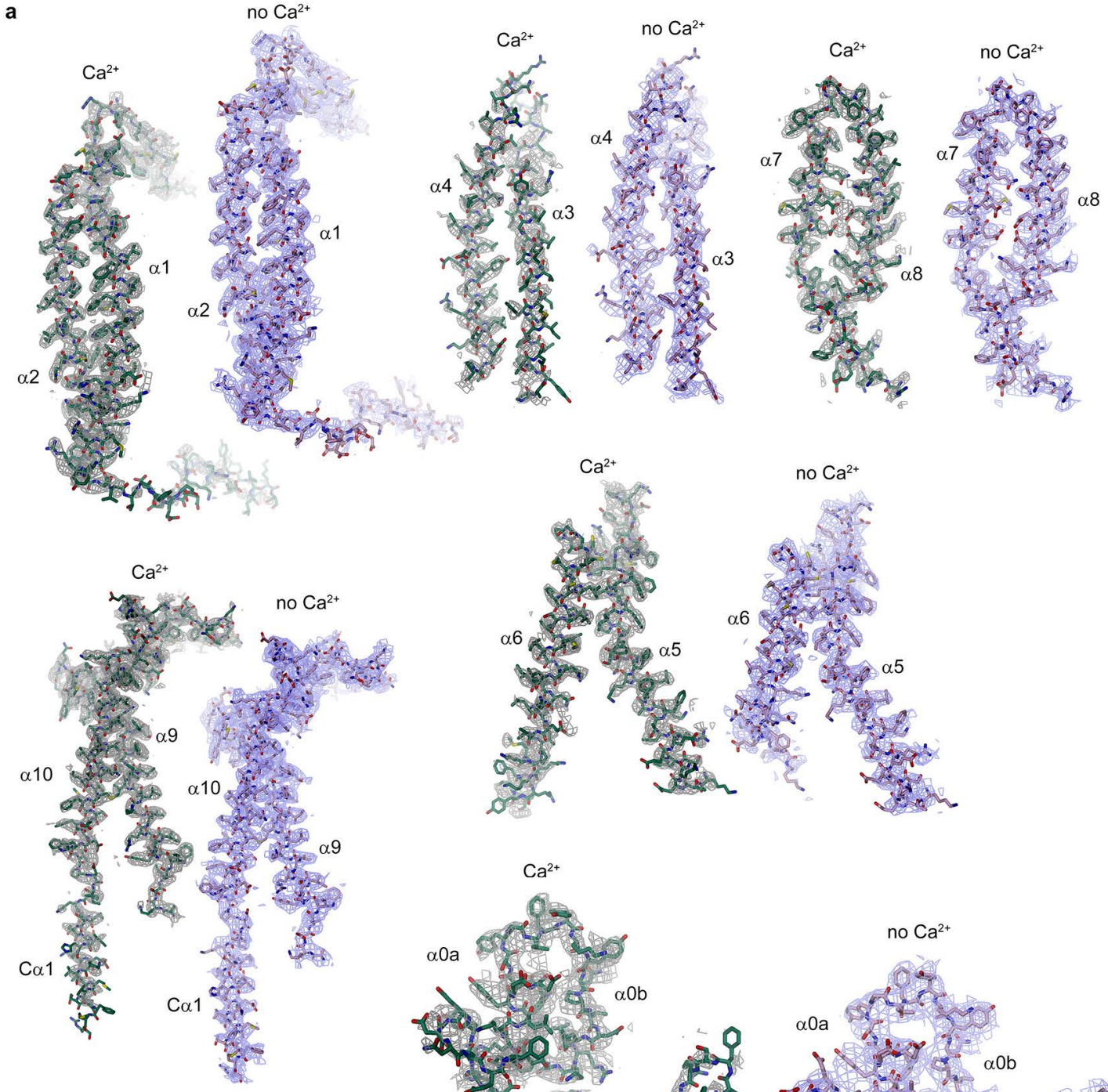

no $\mathrm{Ca}^{2+}$

b
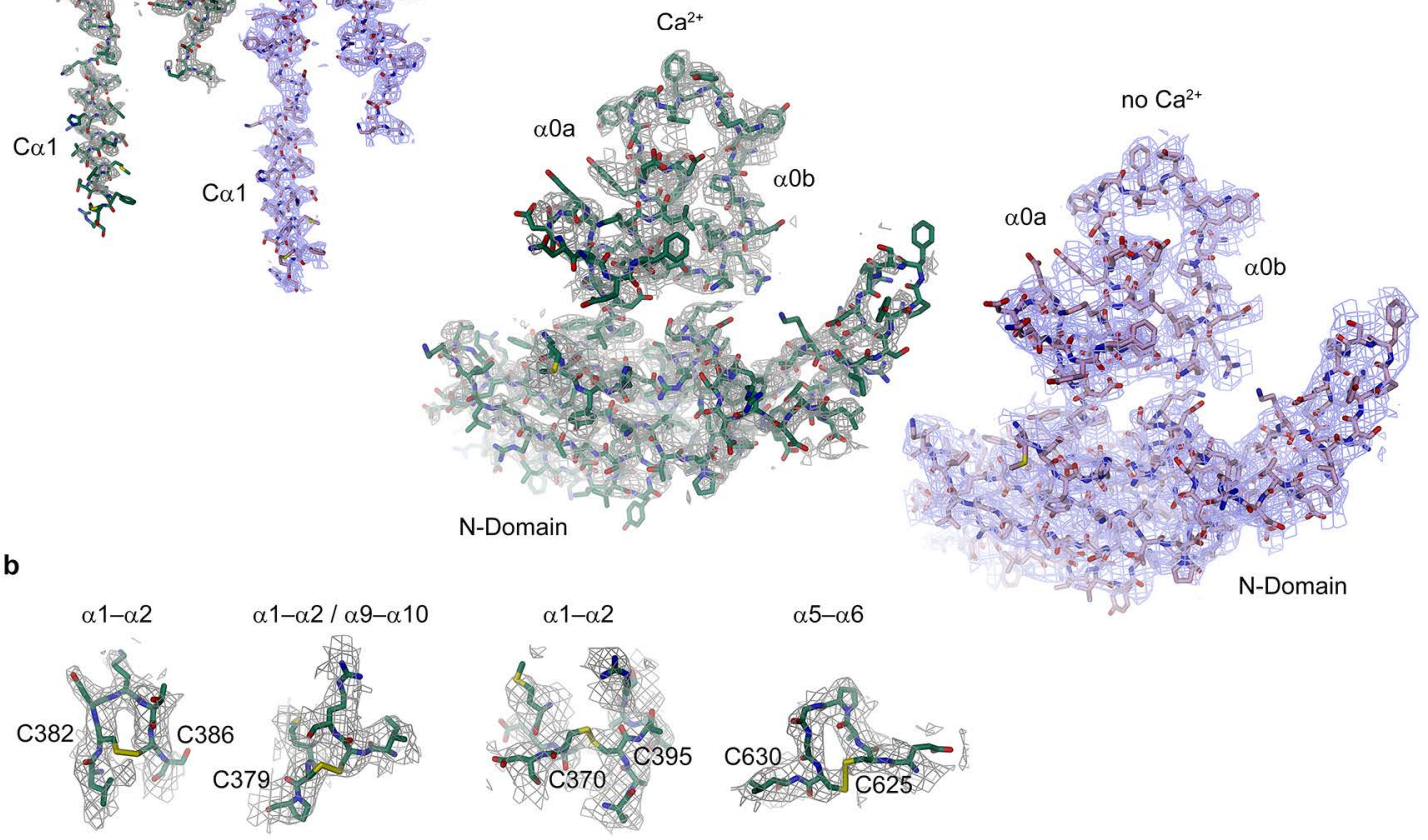

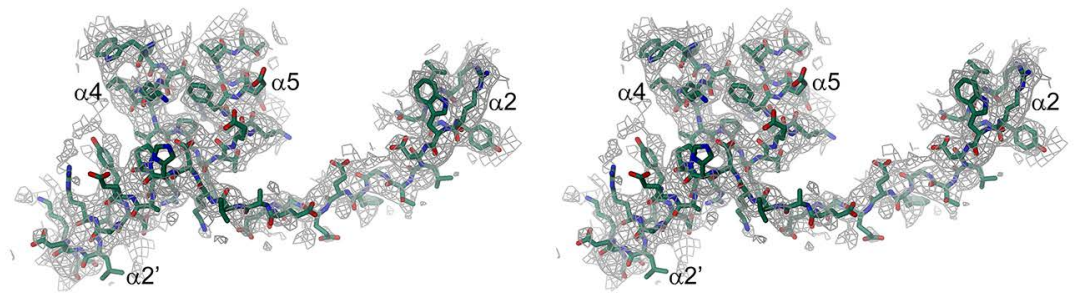

b
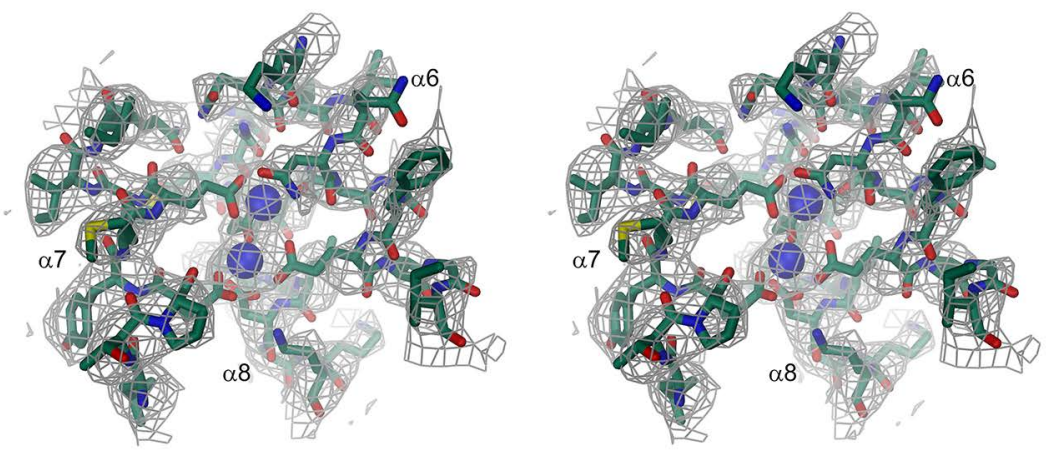

C
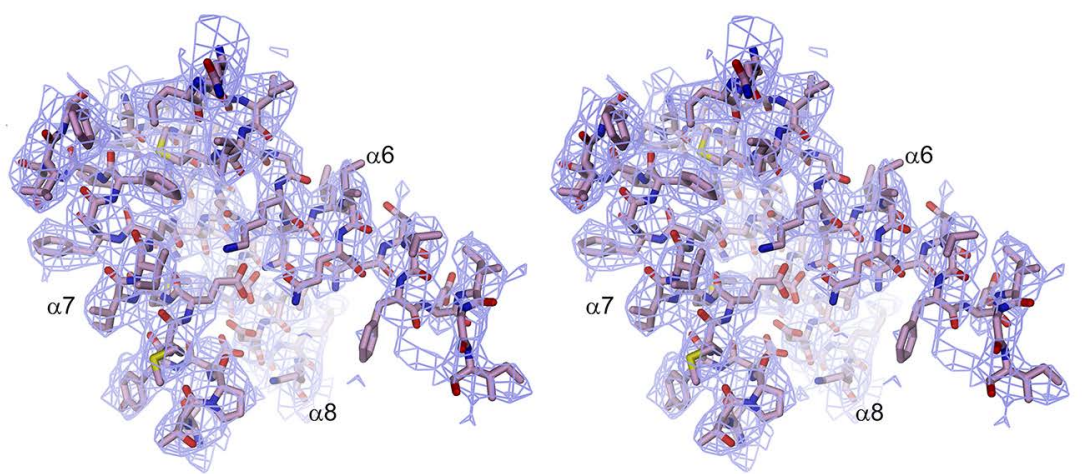

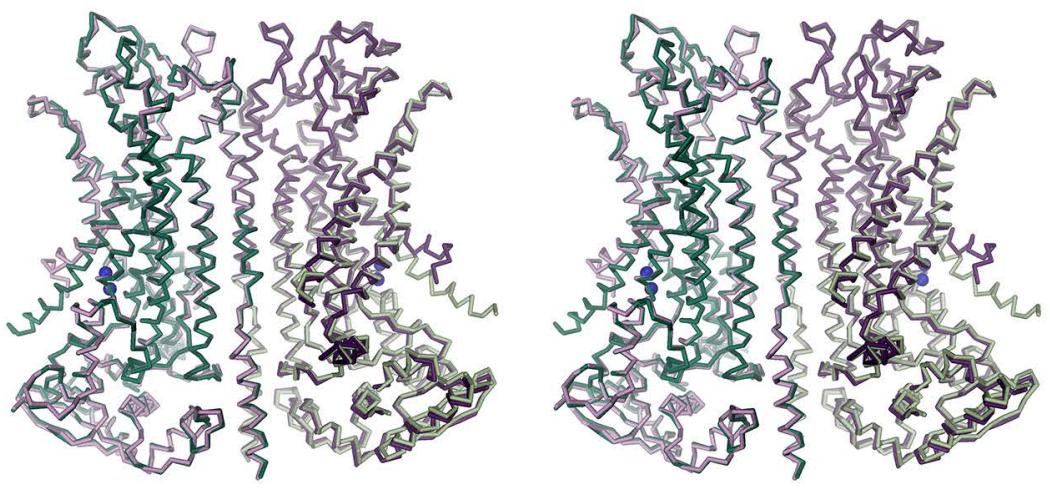

b

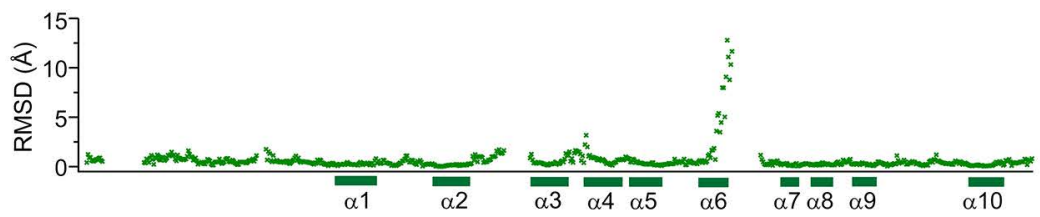

C

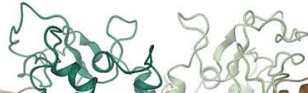

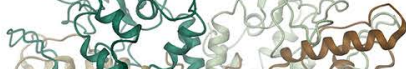

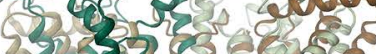

2.)

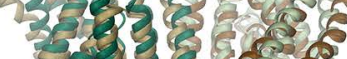

is 1 a ceraso

soly

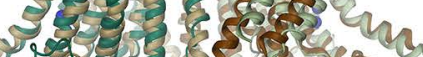

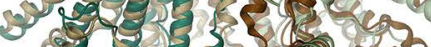

6 Gos

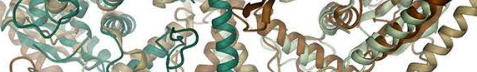
2000

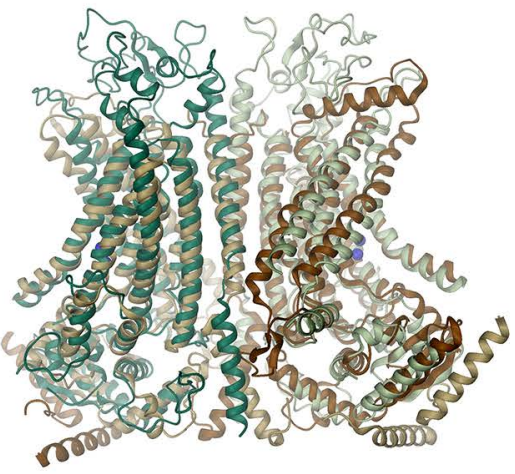

d

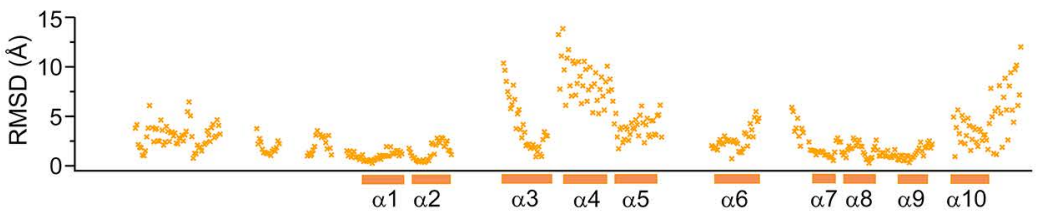




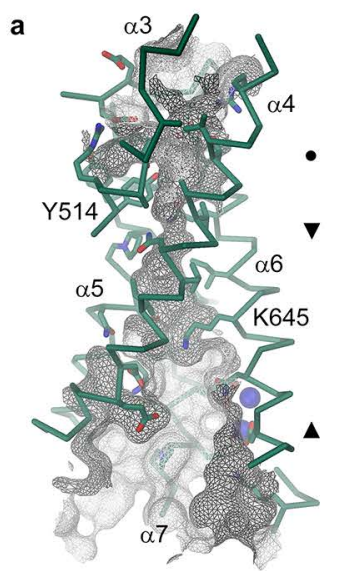

b

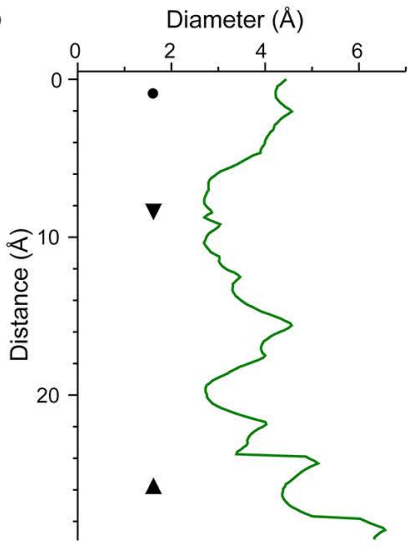

c

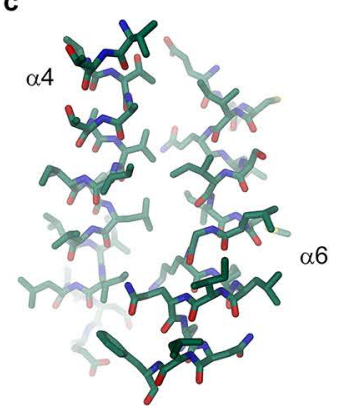

d
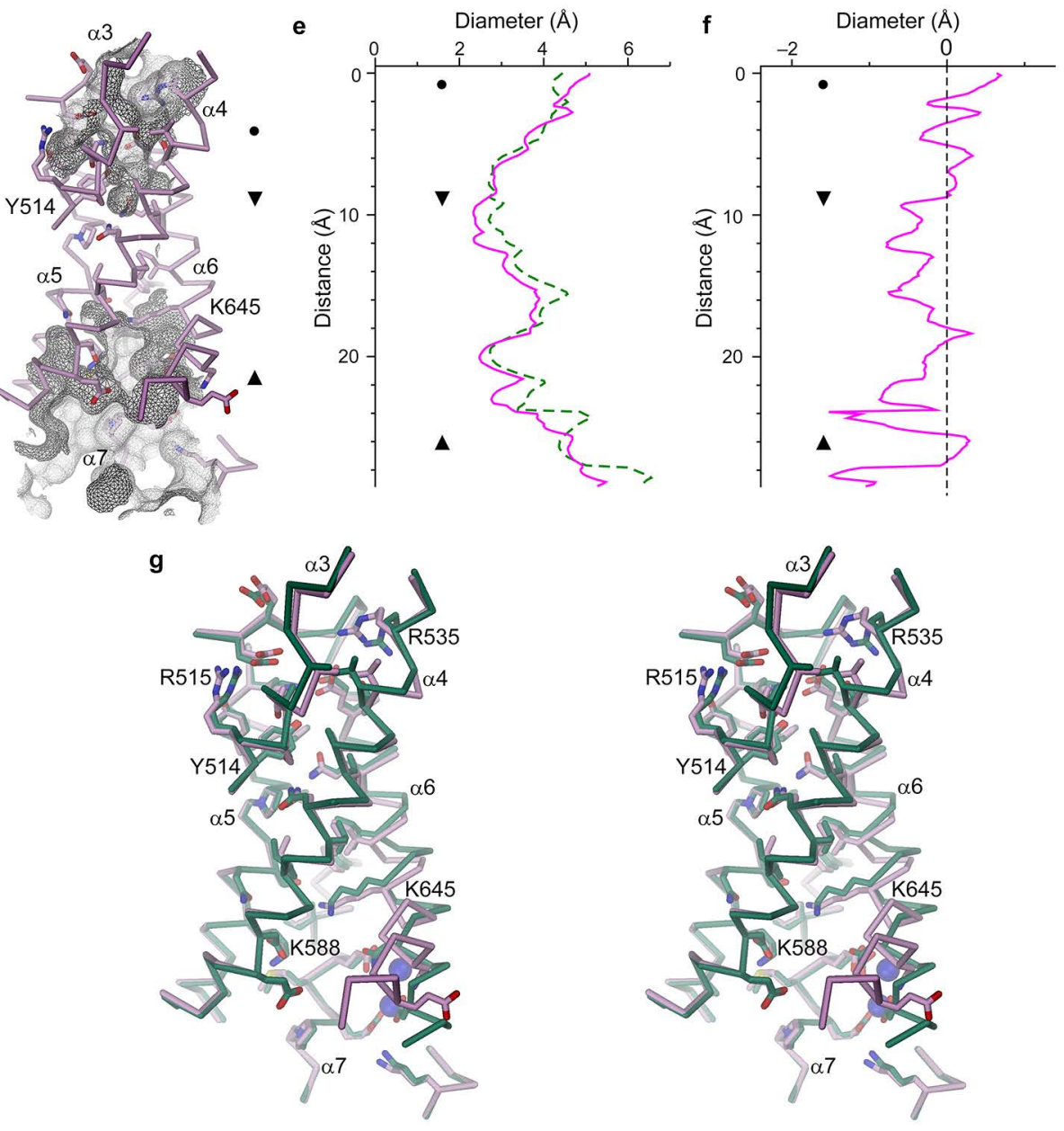
a

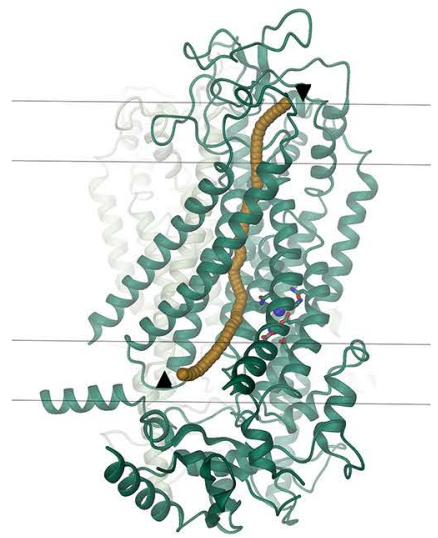

c

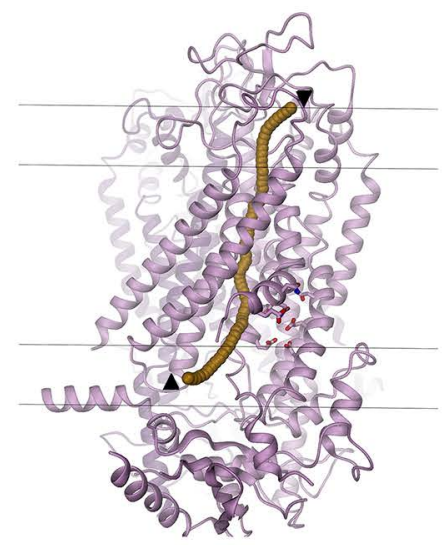

e

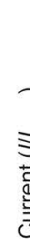

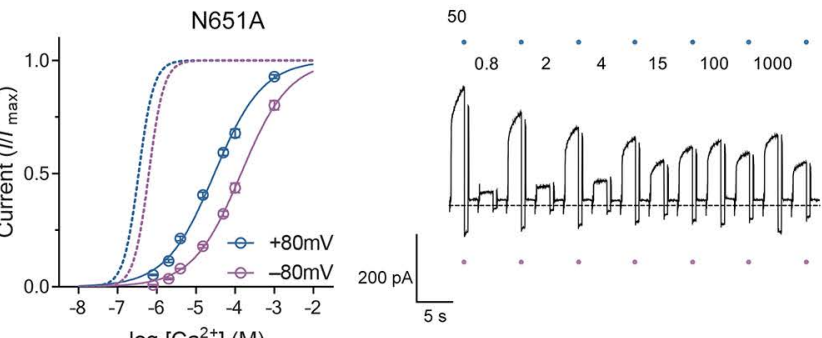

$\log \left[\mathrm{Ca}^{2+}\right](\mathrm{M})$ b
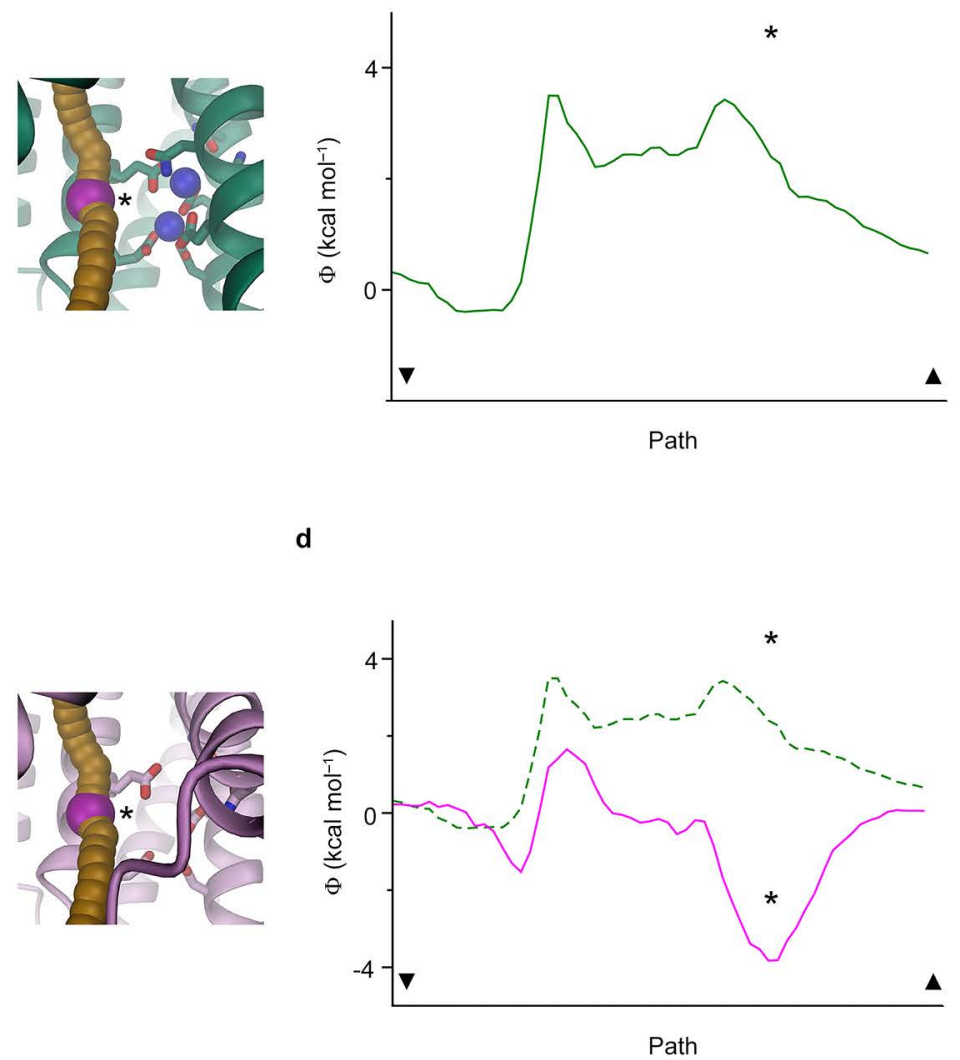

d

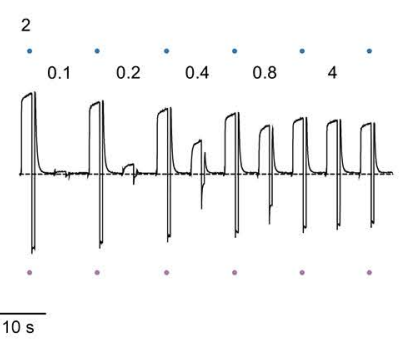

g

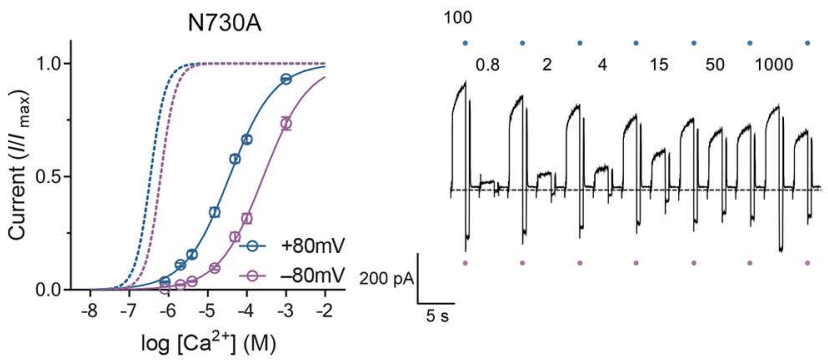




\section{a $\quad \mathrm{G} 644 \mathrm{~A}$}
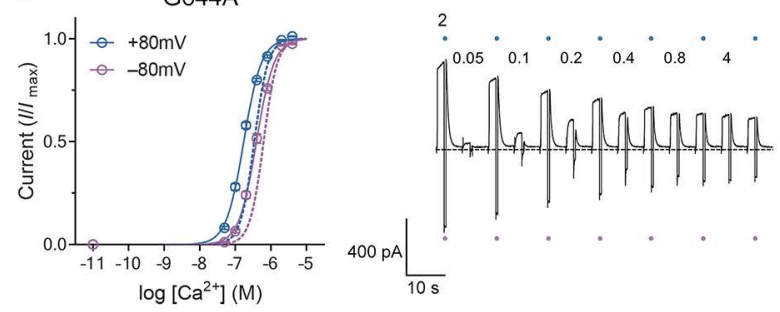

c
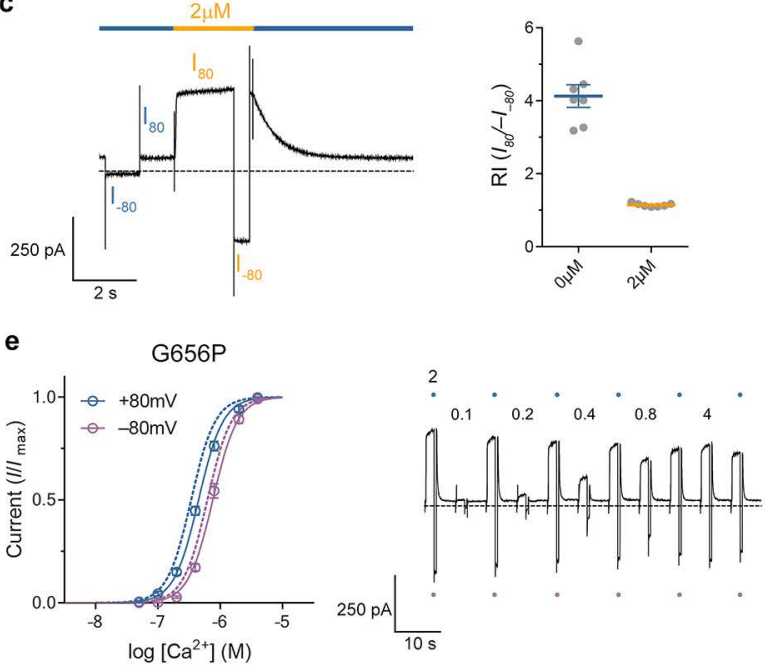

g
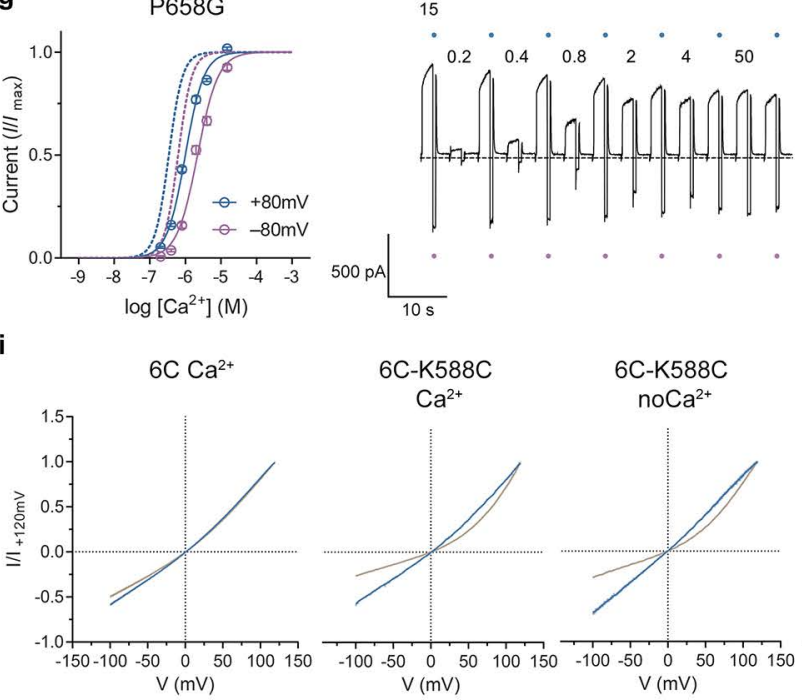

.

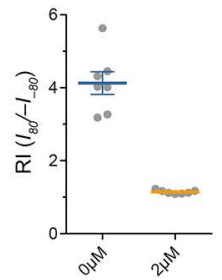

d
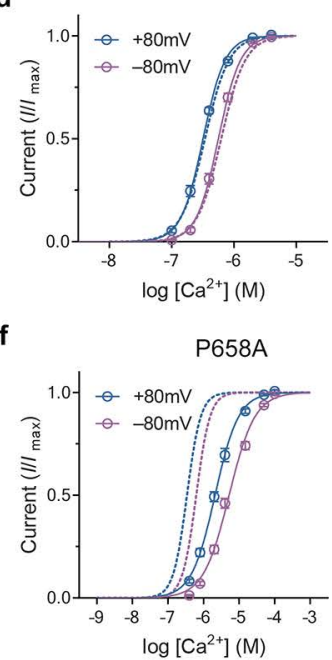

h
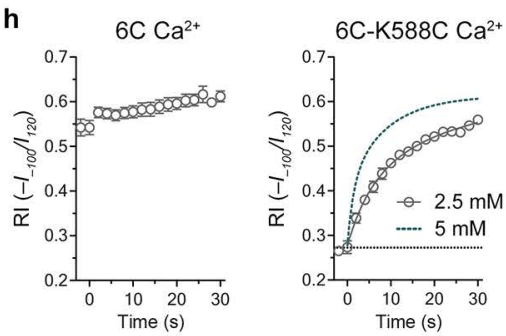

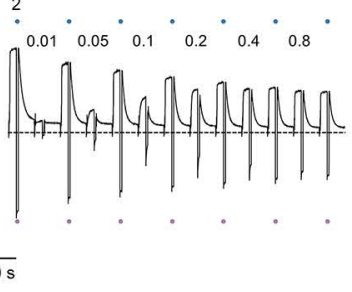

G656A
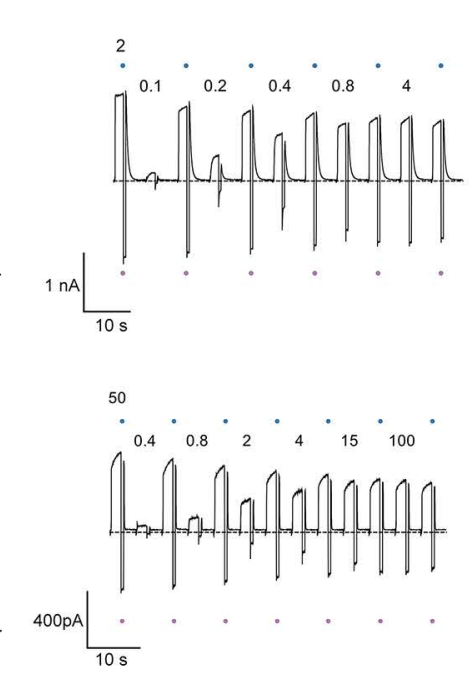

${ }_{0.4}{ }_{0.8}{ }_{0}{ }_{2}{ }^{\circ}{ }_{4}{ }_{15}{ }_{15}{ }_{100}$

6C-K588Q/S592C

j $\mathrm{Ca}^{2+}$
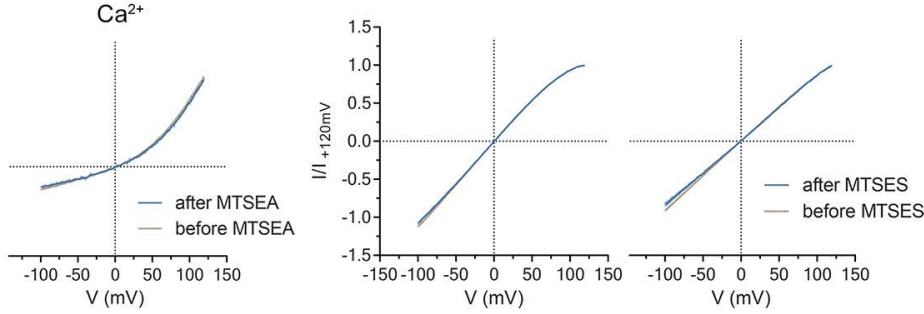

m

WT $\mathrm{Ca}^{2+}$ S592C Ca ${ }^{2+}$

WT $\mathrm{Ca}^{2+}$

$\mathrm{S} 592 \mathrm{C} \mathrm{Ca}^{2+}$

I550A
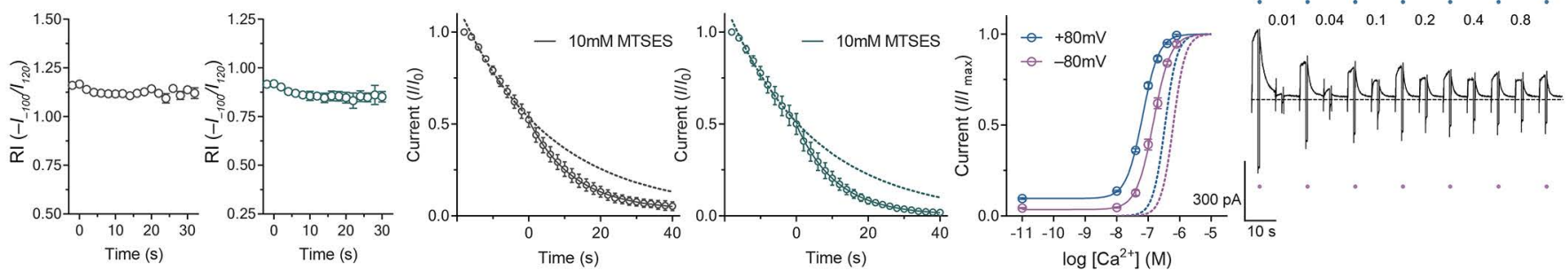
